\title{
A review on Corrosion of High Entropy Alloys: Exploring the Interplay Between Corrosion Properties, Alloy Composition, Passive Film Stability and Materials Selection
}

\author{
Camila Boldrini Nascimento ${ }^{a}$, Uyime Donatus ${ }^{b}$, Carlos Triveño Ríos ${ }^{a}$, \\ Mara Cristina Lopes de Oliveira ${ }^{a}$, Renato Altobelli Antunes ${ }^{a *}$ (])
${ }^{a}$ Universidade Federal do ABC, Centro de Engenharia, Modelagem e Ciências Sociais Aplicadas, Av. Dos Estados, 09210-580, Santo André, SP, Brasil.
${ }^{b}$ Brunel University London, Brunel Centre for Advanced Solidification Technology, Uxbridge, Middlesex UB8 3PH, England.

Received: September 2, 2021; Revised: November 1, 2021; Accepted: December 14, 2021.

\begin{abstract}
The corrosion resistance of high entropy alloys was reviewed in the present work. The main focus was to explore the correlation between alloy composition, passive film stability and corrosion properties and use this information to develop a materials selection procedure based on the Ashby approach. Corrosion current densities and pitting potentials were the main design parameters used to rank different alloys, based on a careful examination of data published in the literature, considering uniform and localized corrosion, respectively. The outputs of the selection process clearly indicated a strong dependence of the corrosion resistance on passive film stability, although microstructural homogeneity and uniform distribution of alloying elements should not be disregarded. Proper combinations of elements such as $\mathrm{Co}, \mathrm{Al}, \mathrm{Ti}, \mathrm{Mo}$ and $\mathrm{Cr}$, especially, are effective at enhancing the corrosion resistance of high entropy alloys.
\end{abstract}

Keywords: High entropy alloys, corrosion, passive film stability, materials selection.

\section{Introduction}

High entropy alloys (HEAs) brought to light a new metallurgical concept in alloy design, breaking the wellestablished approach of developing alloys consisting of only one individual main element as the matrix and minor alloying additions to tailor specific properties ${ }^{1,2}$. The novel design was based on manufacturing alloys with typically five or more main elements whose content ranged between 5 to 35 at. $\%^{3,4}$. The main structural consequence of such innovative concept was the formation of disordered solid solutions during solidification instead of intermetallics ${ }^{5}$. Because of their structural homogeneity, HEAs present an impressive set of attributes. In this panorama, mechanical properties have attracted huge interest for structural engineering applications, owing to the superior ductility, toughness, creep, hardness and fatigue resistance of HEAs when compared to conventional metallic alloys ${ }^{6-8}$. Detailed knowledge regarding the plastic deformation behavior of HEAs is still needed for many systems ${ }^{9}$. Besides mechanical strength, other attractive properties have been explored by several research groups, widening the interest for high entropy alloys. For instance, Chen et al. ${ }^{10}$ prepared a ultrafine-grained $\mathrm{FeCoNiCu}_{0.2} \mathrm{Si}_{0.2} \mathrm{HEA}$ combining excellent mechanical strength and soft magnetic properties. El-Atwani et al. ${ }^{11}$ developed quaternary refractory W-Ta-Cr-V HEA magnetron sputtered films to be used as plasma facing materials in magnetic fusion reactors. The films showed outstanding irradiation resistance due to a favorable crystalline defect mobility in the lattice of the

*e-mail: renato.antunes@ufabc.edu.br
HEA, avoiding the formation of clusters that could lead to irradiation-induced cracks.

Concerns about the corrosion aspects of HEAs are often overridden by their mechanical integrity. However, another major outcome of their compositional homogeneity is related to corrosion stability ${ }^{12,13}$. The corrosion resistance of these complex compositional alloys has been studied by many authors in the past decade. Although the overall homogeneous microstructure comprised of simple solid solutions, HEAs do present some segregation that leads to local chemical heterogeneities, as highlighted by Qiu et al. ${ }^{14}$. Nevertheless, despite the many recent studies devoted to the corrosion behavior of HEAs, several aspects remain unclear. Especial attention must be given to the passive film stability and its relationship with alloy composition ${ }^{15}$. Passivation is a typical characteristic of HEAs, arising due to the common addition of passivating elements such as $\mathrm{Cr}, \mathrm{Ti}, \mathrm{Ni}, \mathrm{Mo}$ and $\mathrm{Al}^{16}$. Each element may influence the alloy structure and passive film composition, giving rise to distinct electrochemical responses, depending on the alloying type and content ${ }^{17,18}$.

Due to the central role played by corrosion resistance in consolidating the future engineering applications of HEAs, this work focuses on exploring the interplay between corrosion properties, passive film stability and alloy composition based on a materials selection standpoint. In the first part of the article, a thorough literature review on the corrosion of HEAs is provided, emphasizing critical issues such as the effect of alloying elements on microstructure and passive film composition and, ultimately, on the corrosion behavior 
of the multicomponent alloys. By critically assessing the information shown in this part, a corrosion-based materials selection of HEAs is discussed in the second part. The materials selection procedure is centered on the Ashby approach ${ }^{19}$. As a well-established methodology, the Ashby method has been successfully employed for selecting materials for a variety of engineering applications, such as micro-electromechanical systems (MEMS), open-cell foams for energy absorption, aircraft materials and automotive body parts ${ }^{20-24}$. The basic steps of this design-driven methodology are: i) translation; ii) screening; iii) ranking; iv) documentation. Details on the characteristics of each stage can be found in the literature ${ }^{25}$. Briefly, in the first stage the design requirements are defined (function, objective, constraints, free variables). The candidates that do not meet the constraints of the translation step are eliminated from the selection process during screening (second step). The remaining candidates are ranked based on the objective(s). Next, additional documentation (not previously considered in the translation step) can be sought by the designer to give further support to the selection results. In the present work, this methodology is unveiled as a tool to identify and rank corrosion-resistant HEAs. Ashby charts are plotted, highlighting straightforward technical criteria for the current and future developments of corrosion-resistant HEAs for structural applications. This information is not available in the current literature, and this will contribute to paving the way towards optimized multi-principal alloy design.

\section{Alloy Composition and Passive Film Stability}

The main alloying elements of HEAs are $\mathrm{Al}, \mathrm{Cr}, \mathrm{Fe}$, $\mathrm{Ni}, \mathrm{Co}, \mathrm{Ti}, \mathrm{Mo}, \mathrm{Mn}$ and $\mathrm{Cu}$. Several other elements may also be encountered such as $\mathrm{Si}, \mathrm{Sn}, \mathrm{V}, \mathrm{C}, \mathrm{N}, \mathrm{W}, \mathrm{Nb}$, Ta and $\mathrm{Zr}$. Versatile alloy design is triggered by this impressive set of possible combinations. As a consequence of this unprecedented design freedom in physical metallurgy, HEA properties can be tailored to meet different purposes. At the core of this scenario, the interplay between alloy composition, microstructure and corrosion resistance has been targeted by many investigators in the past few years. While most studies concentrate on microstructural aspects, passive film composition and stability receives little attention. In the present section, we review the current literature on the corrosion mechanisms of HEAs, giving special emphasis to the correlation between alloy composition and passive film characteristics. It is important to emphasize, though, that either alloy microstructure or processing may profoundly affect the corrosion behavior of high entropy alloys. For instance, if a multiphase microstructure is formed due to specific alloying additions instead of a single solid solution, then galvanic corrosion effects are likely to take place, thus influencing the electrochemical response of the alloy. Some examples are described throughout this part of the paper. Moreover, although its effect is not frequently reported, alloy processing may also influence the corrosion resistance of high entropy alloys ${ }^{13-15}$. Anodizing, laser surface treatments, aging at high temperatures, melting processes, mechanical alloying may lead to different corrosion resistances, depending on the composition of the surface oxide (anodizing), or the formation of heterogeneous microstructures. Phase segregation is a major concern in this case $\mathrm{e}^{14}$. Whenever the processing conditions are a relevant factor for the corrosion behavior of the HEAs included in the present review, its effect will be properly explained, when applicable.

Kao et al. ${ }^{26}$ studied the effect of $\mathrm{Al}$ content on the corrosion resistance of $\mathrm{Al} \mathrm{CoCrFeNi}$ alloy in sulfuric acid solution. Aluminum promoted the formation of an $\mathrm{Al}$ and Ni-rich body centered cubic (BCC) phase in addition to the face centered cubic (FCC) single solid solution that constituted the microstructure of the Al-free alloy. The BCC phase was preferentially corroded upon immersion in the acidic solution, forming a thick and non-protective oxide layer that was linked to soluble complexes of $\mathrm{Al}$ and $\mathrm{Ni}$ easily dissolving in the sulfuric acid medium.

In fact, Shi et al. ${ }^{27}$ observed that the Cr content of $\mathrm{Al}_{\mathrm{x}} \mathrm{CoCrFeNi}$ HEAs decreased in the passive film, as the $\mathrm{Al}$ content increased. The passive film composition was assessed by X-ray photoelectron spectroscopy (XPS), indicating enrichment of $\mathrm{Al}_{2} \mathrm{O}_{3}$ in the passive film and depletion of the passivating $\mathrm{Cr}_{2} \mathrm{O}_{3}$ component. The passive film was thicker and more porous for the Al-containing alloys due to the predominance of $\mathrm{A}_{12} \mathrm{O}_{3}$ over $\mathrm{Cr}_{2} \mathrm{O}_{3}$. The corrosion resistance was, therefore, dependent on the alloy composition and, ultimately, on the passive film composition and density.

Molybdenum may present different effects depending on the electrolyte. Chou et al. ${ }^{28}$ observed that Mo decreased the pitting susceptibility of $\mathrm{Co}_{1.5} \mathrm{CrFeNi}_{1.5} \mathrm{Ti}_{0.5} \mathrm{Mo}_{\mathrm{x}} \mathrm{HEAs}$ in $\mathrm{NaCl}$ solution. Nonetheless, such positive effect depends on the Mo content. If it is increased from 0.1 to $0.8 \mathrm{wt} . \%$ in the base alloy, the formation of Mo and $\mathrm{Cr}$ rich-sigma phase will be promoted at interdendritic regions. At dendritic regions, in turn, Mo and $\mathrm{Cr}$ were depleted, giving rise to an adverse galvanic effect (low surface area ratio between the anodic dendrites and cathodic interdendritic regions). Full characterization of the passive film composition was, however, not reported. In a recent publication, Dai et al. ${ }^{29}$ shed light on this topic. They studied the effect Mo content on the corrosion behavior of $\mathrm{FeCoCrNiMo}_{\mathrm{x}}$. As also indicated by Chou et al. ${ }^{28}$, the corrosion resistance was dependent on the Mo content. For $0.1 \mathrm{wt} . \%$ Mo, the passive film consisted of a $\mathrm{Cr}_{2} \mathrm{O}_{3}$ rich film with the incorporation of Mo oxides, increasing its stability and corrosion resistance. However, higher amounts of molybdenum led to the formation of galvanic couples between the matrix (depleted in $\mathrm{Cr}$ and $\mathrm{Mo}$ ) and Mo and Cr-rich precipitates. Hence, if a multi-phase alloy is formed, galvanic effects may hamper the corrosion resistance of the HEA. Shang et al. ${ }^{30}$ confirmed this observation for $\mathrm{CoCrFeNiMo}_{\mathrm{x}}(\mathrm{x}=0.1$ to 0.5$)$ alloys in sulfuric acid and sodium chloride solutions. For $\mathrm{x}=0.3$ to 0.5 precipitation of a secondary- $\sigma$ phase inside interdendrites was detrimental to the corrosion resistance due to a galvanic coupling effect between $(\mathrm{Mo}, \mathrm{Cr})$-rich and $(\mathrm{Mo}, \mathrm{Cr})$-depleted regions around the $\sigma$ phase.

Chromium is part of several traditional multicomponent alloys. As its passivating character is well-known for conventional iron-based alloys, it is plausible to hypothesize that a similar effect would also take place for HEAs. Confirming the prominent role of chromium in the corrosion resistance of HEAs, Chai et al. ${ }^{31}$ studied the electrochemical 
behavior of $\mathrm{FeCoNiCr}_{\mathrm{x}}(\mathrm{x}=0,0.5,1.0) \mathrm{HEAs}$ in $\mathrm{H}_{2} \mathrm{SO}_{4}$ and $\mathrm{NaCl}$ solutions. The corrosion resistance of the alloy was dependent on the $\mathrm{Cr}$ content. The highest $\mathrm{Cr}$ content entails its segregation at interdendritic regions, thus leading to severe localized corrosion at these regions. By contrast, the intermediate $\mathrm{Cr}$ content promoted the formation of a highly corrosion-resistant passive film, mainly composed of $\mathrm{Cr}_{2} \mathrm{O}_{3}$. In the absence of chromium, the passive film is comprised of $\mathrm{Fe}_{2} \mathrm{O}_{3}, \mathrm{NiO}$ and $\mathrm{CoO}$ that were less stable in the acidic electrolyte, dissolving at a much faster rate when compared to the Cr-rich passive film of the $\mathrm{FeCoNiCr}_{0.5}$ alloy. From these results, it is noteworthy that there is optimum chromium content to achieve the best corrosion resistance in the $\mathrm{FeCoNiCr}$ system. Chen et al. ${ }^{32}$ studied the effect of chromium addition on the corrosion resistance of $\left(\mathrm{CuFeNiMn}_{1-\mathrm{x}}\right)$ $\mathrm{Cr}_{\mathrm{x}} \operatorname{HEAs}(\mathrm{x}=0,0.05,0.10,0.15,0.20$ and 0.25$)$ in nitric acid solution. The corrosion rate depends on the chromium content. Element segregation at dendritic and interdendritic regions is associated with the different electrochemical responses of the alloys. $\mathrm{Cu}$ and $\mathrm{Mn}$ were segregated in the interdendritic regions, forming a stable FCC phase. $\mathrm{Cr}, \mathrm{Fe}$ and $\mathrm{Ni}$ were mainly segregated at dendritic regions, also forming an FCC phase. As the Cr content reaches 15\% an additional BCC phase is formed in the dendritic regions, wherein $\mathrm{Cr}$ is also segregated. Such elemental distribution gave rise to galvanic effects between $\mathrm{Cu}$-rich interdendritic and $\mathrm{Cr}$-rich dendritic regions, triggering the corrosion process of the HEAs. As the volume fraction of the Cr-rich BCC dendritic phase increased with the $\mathrm{Cr}$ content, so did the corrosion resistance of the alloy. The $\mathrm{Cu}$-rich interdendritic region is the anode in this galvanic cell, being preferentially corroded. Alloy microstructure was, therefore, an important factor for the corrosion behavior of the $\left(\mathrm{CuFeNiMn}_{1-x}\right) \mathrm{Cr}_{x}$ HEAs. The report by Parakh et al..$^{33}$ confirmed the relevant role played by alloy microstructure for the corrosion behavior of AlNiCoCrFe HEA in sodium chloride solution. This alloy presented a mixed $\mathrm{FCC}$ and $\mathrm{BCC}$ structure. $\mathrm{Cr}$ and $\mathrm{Al}$ were enriched in the BCC phase, whereas $\mathrm{Ni}, \mathrm{Co}$ and Fe were enriched in the FCC phase. They mention two competing effects related to the chemical stability of the FCC phase. As this phase is more compact than the BCC phase, the energy to remove an atom from its surface is higher, and there is a trend of forming non-porous oxide layers. This, in turn, would increase the corrosion resistance of an FCCrich alloy. Conversely, the FCC phase is depleted in the passivating elements $\mathrm{Cr}$ and $\mathrm{Al}$, and richer in the more easily corroded $\mathrm{Ni}, \mathrm{Fe}$ and $\mathrm{Co}$ elements. Thus, it is expected that higher FCC volume fractions would decrease the corrosion resistance of the alloy. In this respect, there should be an optimum balance between the two phases in order to achieve high corrosion resistance.

However, for other systems, other trends can be found. For instance, Yang et al. ${ }^{34}$ prepared $\mathrm{AlCr}_{\mathrm{x}} \mathrm{FeNi}_{2} \mathrm{Cu}_{1.6} \mathrm{HEAs}$ and investigated the effect of the $\mathrm{Cr}$ content on the corrosion resistance in $3.5 \mathrm{wt} . \% \mathrm{NaCl}$ solution. The surface chemical stability was unequivocally enhanced as the $\mathrm{Cr}$ content increased. The structure of the alloy was not significantly altered by the chromium content, being comprised of a mixture of FCC and BCC phases at dendritic and interdendritic regions, respectively. Distribution of elements in each phase and the overall passive film composition should affect the corrosion resistance of the alloys. However, passive film composition was not assessed in detail. In a recent publication, Yan et al. ${ }^{35}$ have drawn similar conclusions on the effect of $\mathrm{Cr}$ on the corrosion behavior of $\mathrm{Al}_{0.3} \mathrm{Cr}_{x} \mathrm{FeCoNi}$ high entropy alloys. The corrosion resistance increased with the $\mathrm{Cr}$ content in the alloy, mainly due to $\mathrm{Cr}_{2} \mathrm{O}_{3}$-enrichment of the passive film and not due to microstructural aspects of the different Cr-containing alloys. Based on XPS analysis of the passive film, they observed an increase in $\mathrm{Cr}^{3+}$ as the chromium content was increased. The report by Wang et al. ${ }^{36}$ reveals a similar trend for the passive film of an equiatomic $\mathrm{CoCrFeMnNi} \mathrm{HEA} \mathrm{formed} \mathrm{in} \mathrm{acidic} \mathrm{solution.} \mathrm{Chromium}$ was enriched in the oxide layer due to its slow dissolution rate when compared to iron. Another interesting aspect is the predominance of $\mathrm{Cr}_{2} \mathrm{O}_{3}$ as the oxide phase in the passive film, surpassing the concentrations of $\mathrm{Fe}, \mathrm{Mn}$ and Co oxides, while Ni oxide was not detected. The high thermodynamic stability of $\mathrm{Cr}_{2} \mathrm{O}_{3}$ would explain the prevalence of $\mathrm{Cr}^{3+}$ ions in the passive film. Han et al. ${ }^{37}$ evidenced the central role played by Cr-depletion on the corrosion behavior of ultra-fine grained CoNiFeCrMn HEAs. Cr-depleted regions were formed in the ultra-fine grained alloy whereas a more homogeneous chromium element distribution was observed for the conventional coarse-grained material. Enhanced diffusion of point defects such as oxygen vacancies and cation vacancies through the passive film formed on the ultra-fine grained material would occur easily in Cr-depleted regions, thus reducing the stability of the passive film. Based on the reports of such varying effects of $\mathrm{Cr}$ addition for different HEA systems, Fu et al. ${ }^{13}$ highlighted the need for new investigations that take into account the synergism between chromium and different alloying elements with potential influence on the corrosion properties of HEAs.

Nickel is another common alloying element of HEAs. Nickel content was found to affect the microstructure and, ultimately, the hardness of $\mathrm{VCrMnFeCoNi}_{x}$ and $\mathrm{CrFeMnCoNi}_{x}$ alloys $^{38,39}$. In both systems, the hardness decreased as the nickel content increased. The formation of a single FCC phase for the alloys with high Ni content (> 10 at.\%) was responsible for the "softer" character of the $\mathrm{CrFeMnCoNi}{ }_{x}$ alloys. Regarding the $\mathrm{VCrMnFeCoNi} \mathrm{x}_{\mathrm{x}}$ alloys, hardness decrease was associated with the decrease of the concentration of the sigma-phase (matrix) and the formation of a dualphase microstructure consisting a mixture of sigma and FCC phases (composite structure). The plastic character of the alloy was, therefore, dependent on the relative amount of each one of these phases. Despite its well-known effect on the microstructure and mechanical properties of some multicomponent alloys, the relationship between the nickel content and the corrosion resistance of HEAs is not frequently reported. Qiu and $\mathrm{Liu}^{40}$ gave a contribution towards the understanding of the role of $\mathrm{Ni}$ on the corrosion properties of $\mathrm{Al}_{2} \mathrm{CrFeCoCuTiNi}_{\mathrm{x}} \mathrm{HEAs}$ prepared by laser cladding. The corrosion resistance was improved up to $\mathrm{x}=1.0$. Conversely, by further increasing the Ni molar content, the corrosion current density increased, accelerating the corrosion rate. Such effect was explained based on microstructural aspects. However, details regarding the passive film composition were missing, and the corrosion mechanism was not clearly 
described. More recently, Ríos et al. ${ }^{41}$ investigated the effect of the nickel content on the corrosion behavior of $\mathrm{AlCoCrFeNi} i_{x}$ alloys. Similarly to what was reported by Qiu and $\mathrm{Liu}^{40}$, they observed that the corrosion resistance did not follow a linear relationship with the Ni content. Such varying trend was hypothesized to be originated from the possible predominance of other influencing factors such as alloy microstructure and distribution of alloying elements over the constituent phases. This statement is supported by the results. Nonetheless, this complex interaction was not addressed in the text. Huang et al. ${ }^{42}$ studied the corrosion behavior of CoCrNiAlTi HEAs with different nickel contents in sulfuric acid solution. They observed that nickel does not increase the stability of the passive film, acting as a hydrolysis promoter for $\mathrm{Cr}^{3+}$ ions, thus decreasing the corrosion resistance of the alloy.

The effect of cobalt concentration in the corrosion resistance of HEAs has received little attention in the literature. Qiu ${ }^{43} \mathrm{did}$ not find a clear connection between the Co content and the corrosion resistance of $\mathrm{Al}_{2} \mathrm{CrFeCo}_{\mathrm{x}} \mathrm{CuNiTi} \mathrm{HEA}$ coating either in alkaline or sodium chloride solutions. Zhao et al. ${ }^{44}$, in turn, reported an increase in the passive film stability of sintered $\mathrm{Co}_{\mathrm{x}} \mathrm{CrCuFeMnNi}$ HEAs. According to the authors, such result arises from two factors. Firstly, as the content of passivating elements (such as cobalt) increases, so does the passive film stability. Secondly, Co triggers the stability of $\mathrm{Cr}_{2} \mathrm{O}_{3}$ and $\mathrm{Ni}(\mathrm{OH})_{2}$-rich passive films as an adsorption layer of $\mathrm{Co}(\mathrm{OH})_{2}$ is formed. However, full characterization of the passive film composition was not shown. Additionally, the authors reckoned that corrosion resistance would be also determined by alloy's microstructure which, in turn, depends on the Co concentration in the alloy. In fact, the alloys were comprised of two FCC phases. The first one (FCC1) was $(\mathrm{Fe}, \mathrm{Cr})$-rich while the second one $(\mathrm{FCC} 2)$ was $(\mathrm{Co}, \mathrm{Cu}$, $\mathrm{Ni}, \mathrm{Mn}$ )-rich. The relative concentration of the Co-rich FCC2 phase increased with the Co content, and so did the corrosion resistance of the HEA. Electrochemical impedance spectroscopy measurements suggested that the passive film thickness increased with the Co content, thus enhancing its protective character against corrosion.

Recently, Wu et al. ${ }^{45}$ evaluated the corrosion mechanism of a non-equiatomic FeNiCoCr alloy $\left(\mathrm{Fe}_{40} \mathrm{Ni}_{20} \mathrm{Co}_{20} \mathrm{Cr}_{20}\right)$ in $0.1 \mathrm{M} \mathrm{H}_{2} \mathrm{SO}_{4}$ solution at room temperature. The corrosion resistance was found to be higher than that of conventional $316 \mathrm{~L}$ stainless steel. The explanation for the improved performance against corrosion was attributed to the composition and stability of the passive film. One aspect is related to the high $\mathrm{Cr} / \mathrm{Fe}$ ratio in the passive film of the HEA. As reported in the literature, Ni hinders the access of $\mathrm{Fe}$ to the passive film, facilitating the increase of chromium concentration with respect to iron. An interesting aspect in the passivation process of the HEA is the role played by Co. Based on XPS results, the authors proposed that $\mathrm{Co}$ ions tend to enter the lattice of $\mathrm{Cr}_{2} \mathrm{O}_{3}$ or $\mathrm{Fe}_{2} \mathrm{O}_{3}$, replacing trivalent $\mathrm{Cr}$ and $\mathrm{Fe}$ ions at octahedral positions, thus forming mixed oxides such as $\mathrm{CoCr}_{2} \mathrm{O}_{4}$ and $\mathrm{CoFe}_{2} \mathrm{O}_{4}$. As a consequence of the relative dense structure of Co-doped oxides, the passive film tends to be more compact than that of a $\mathrm{Co}$-free oxide layer, increasing the passive film stability. One further aspect that enhances the passive film stability of the HEA is the presence of bound water that helps in the healing of the oxide layer in case it breaks, preventing corrosion.

Manganese is one of the main alloying elements in many HEA systems due to its effect in the phase stability and mechanical properties. Thus, its role in the passivation processes of these multiprincipal component alloys is worth investigating. Hence, the ramifications of alloy composition on the corrosion resistance of HEAs were subject of studies devoted to understanding the effect of the manganese content. Torbati-Sarraf et al. ${ }^{46}$ gave an important contribution on this topic by studying the effect of $\mathrm{Mn}$ incorporation on the pitting corrosion of $\mathrm{CrFeCoNi}$ HEAs at different temperatures. The Mnfree alloy was less prone to pitting than the Mn-containing one. XPS analyses of the passive films were effective at revealing that $\mathrm{Mn}$ addition depleted the $\mathrm{Cr}$ compounds in the passive film, thus increasing its dissolution rate, and reducing the pitting corrosion potentials. Similar conclusions were drawn by Wong et al. ${ }^{47}$ for $\mathrm{Al}_{03} \mathrm{CoCrFeNiMn}$. HEAs. Passivating chromium and nickel compounds were depleted as the Mn content increased, thus hampering the corrosion protection ability of the oxide film. The deleterious effect of $\mathrm{Mn}$ on the corrosion resistance of conventional metallic alloys is documented in the literature. Manganese oxide is found to be unstable in Fe-Mn alloys ${ }^{48}$. Sahu et al ${ }^{49}$ have confirmed the connection between manganese and pitting corrosion susceptibility of NiFeCrMnCo non-equiatomic HEAs.

Copper addition is reported to boost the corrosion resistance of $\mathrm{HEAs}^{10}$. Abolkassem et al..$^{50}$ assessed the corrosion resistance of $\mathrm{FeNiCoCr}$ HEA with $\mathrm{Cu}$ addition in acidic solutions. The alloys were prepared by powder metallurgy. Based on the characterization of the corrosion behavior of the alloys, the $\mathrm{Cu}$-containing HEA was less prone to corrosion than the FeNiCoCr-base alloy. Homogeneous distribution of passivating elements in the passive film (including $\mathrm{Cu}$ ) is assumed as the central cause of the high corrosion resistance of the $\mathrm{Cu}$-containing alloy. Even though these results point to a beneficial effect of $\mathrm{Cu}$ on the corrosion resistance of the HEA, one should not neglect the possible detrimental nature of $\mathrm{Cu}$ segregations. The relevance of avoiding $\mathrm{Cu}$ segregation in the $\mathrm{CoCrFeNiCu}$ system has been recognized by Zheng et al. ${ }^{51}$. The extensive segregation at grain boundaries or interdendritic regions arises from high mixing enthalpies between $\mathrm{Cu}$ and $\mathrm{Co}, \mathrm{Cr}$ and $\mathrm{Fe}^{52}$. These authors focused on the relationship between $\mathrm{Cu}$ segregation, microstructure and mechanical properties of $\mathrm{CoCrFeNiCu} \mathrm{HEA}$. Other researchers have also explored the interplay between $\mathrm{Cu}$ segregation and the electrochemical behavior of the HEA. Nene et al. ${ }^{53}$ gave a clear picture of the prominent role played by $\mathrm{Cu}$ segregation on the corrosion behavior of vacuum arccast $\mathrm{Fe}_{38.5} \mathrm{Mn}_{20} \mathrm{Co}_{20} \mathrm{Cr}_{15} \mathrm{Si}_{5} \mathrm{Cu}_{1.5}$ HEA. $\mathrm{Cu}$ segregation was found to control the corrosion response in sodium chloride solution. Non-uniform pitting corrosion was observed for the $\mathrm{Cu}$-containing alloy due to $\mathrm{Cu}$ segregation at specific sites. By eliminating segregation through a friction stir processing, the number of pits was significantly reduced, as well as pitting susceptibility. Further explanation of the corrosion mechanism was based on the lower passivation tendency of the alloy in the presence of $\mathrm{Cu}$ segregations, as the passive film becomes less stable due to microgalvanic 
cells formed between the alloy matrix (depleted in $\mathrm{Cu}$ ) and the $\mathrm{Cu}$-rich regions.

Scully et al. ${ }^{54}$ gave an excellent overview to drive the development of corrosion resistant multi-principal alloys. The importance of selecting alloying elements that enable the formation of protective oxide films was highlighted. As a rule-of-thumb for successful design against corrosion, some points were pinpointed such as the passivation ability of the chosen element, thus forming a prominent part of the oxide, adding dopants that are known to enhance the passivation stability of the dominant oxide, and choosing elements that are capable of forming a new oxide compound.

\section{Corrosion Properties}

In the previous section, fundamental aspects of the complex relationship between microstructure, chemical composition, stability of the passive film and the corrosion behavior of HEAs were reviewed. In the past few years, many researchers have developed huge variety of new multiprincipal element alloys, exploring different compositions and properties. Alloy composition has an obvious importance in this regard, determining the most important structural features and deeply affecting the passive film stability. The main goal of the present section is to provide the reader a detailed panorama of the current literature with respect to alloy composition, structure and corrosion properties of HEAs. Based on a thorough assessment of literature reports, relevant data were obtained for different materials, as displayed in Tables 1 and 2, for results obtained in acidic and sodium chloride solutions, respectively. Corrosion potential $\left(\mathrm{E}_{\text {corr }}\right)$ and corrosion current density $\left(i_{\text {corr }}\right)$ values are presented. Pitting potentials $\left(\mathrm{E}_{\mathrm{pit}}\right)$ and passive current densities $\left(\mathrm{i}_{\text {pass }}\right)$ are also shown, when available. All potentials were converted to the saturated calomel electrode (SCE) unit. Electrolyte

Table 1. Alloy composition, structure and corrosion properties of different HEAs. Data obtained in acidic solutions.

\begin{tabular}{|c|c|c|c|c|c|c|c|}
\hline Alloy composition & Structure & $\begin{array}{c}\mathrm{E}_{\text {corr }} \\
\left(\mathrm{mV}_{\mathrm{SCE}}\right)\end{array}$ & $\begin{array}{c}\mathrm{i}_{\text {corr }}(\mu \mathrm{A} . \\
\left.\mathrm{cm}^{-2}\right)\end{array}$ & $\begin{array}{c}\mathrm{i}_{\text {pass }}(\mu \mathrm{A} . \\
\left.\mathrm{cm}^{-2}\right)\end{array}$ & $\begin{array}{c}\mathrm{E}_{\mathrm{pit}} \\
\left(\mathrm{mV}_{\mathrm{SCE}}\right)\end{array}$ & $\begin{array}{l}\text { Additional } \\
\text { comments }\end{array}$ & Reference \\
\hline $\mathrm{CoCrFeNi}$ & FCC & 161 & 15.8 & 4.5 & 244 & \multirow{2}{*}{$\begin{array}{c}\text { Electrolyte: } 0.5 \\
\text { mol.L-1 } \mathrm{H}_{2} \mathrm{SO}_{4} \\
\text { solution at RT }\end{array}$} & 26-a \\
\hline $\mathrm{AlCoCrFeNi}$ & $\mathrm{FCC}+\mathrm{BCC}$ & 148 & 13.1 & 13.9 & 252 & & 26-b \\
\hline $\mathrm{Co}_{1.5} \mathrm{CrFeNi}_{1.5} \mathrm{Ti}_{0.5}$ & FCC & 150 & 30 & 9 & 1089 & $\begin{array}{l}\text { Interdendrites } \\
\text { rich in Ti and } \mathrm{Ni} \text {; } \\
\text { dendrites rich } \\
\text { in } \mathrm{Fe} \text { and } \mathrm{Cr} ; \\
\text { Electrolyte: } 0.5 \\
\text { mol. } \mathrm{L}^{-1} \mathrm{H}_{2} \mathrm{SO}_{4} \\
\text { solution at RT }\end{array}$ & 28-a \\
\hline $\mathrm{Co}_{1.5} \mathrm{CrFeNi}_{1.5} \mathrm{Ti}_{0.5} \mathrm{Mo}_{0.8}$ & $\begin{array}{l}\mathrm{FCC}+\mathrm{Cr} \text { and Mo- } \\
\text { rich sigma phase }\end{array}$ & 172 & 69 & 22 & 1089 & $\begin{array}{l}\text { Interdendrites } \\
\text { rich in } \mathrm{Cr} \text { and } \\
\mathrm{Mo} \text {; dendrites } \\
\text { rich in Co and } \mathrm{Ni} \text {; } \\
\text { Electrolyte: } 0.5 \\
\text { mol. } \mathrm{L}^{-1} \mathrm{H}_{2} \mathrm{SO}_{4} \\
\text { solution at RT }\end{array}$ & 28-b \\
\hline $\mathrm{CoCrFeNiMo}_{0.4}$ & $\begin{array}{l}\mathrm{FCC}+\mathrm{Cr} \text { and Mo- } \\
\text { rich sigma phase }\end{array}$ & -289 & 0.712 & 6 & 88.5 & $\begin{array}{l}\text { Electrolyte: } 0.5 \\
\text { mol.L }{ }^{-1} \mathrm{H}_{2} \mathrm{SO}_{4} \\
\text { solution at RT }\end{array}$ & 30 \\
\hline $\mathrm{CoCr}_{2} \mathrm{Ni}_{2} \mathrm{AlTi}$ & $\begin{array}{c}\text { Two BCC phases + } \\
\mathrm{Al}_{x} \mathrm{Co} \\
\end{array}$ & -240 & 7.24 & 2.45 & 886 & \multirow{3}{*}{$\begin{array}{l}\text { Electrolyte: } 0.5 \\
\text { mol.L } \mathrm{L}^{-1} \mathrm{H}_{2} \mathrm{SO}_{4} \\
\text { solution at RT }\end{array}$} & 42-a \\
\hline $\mathrm{Co}_{2} \mathrm{Cr}_{2} \mathrm{Ni}_{2} \mathrm{AlTi}$ & $\begin{array}{c}\mathrm{FCC}+\mathrm{BCC}+ \\
\mathrm{NiCoCr}+\mathrm{Al}_{x} \mathrm{Co} \\
\end{array}$ & 107 & 0.654 & 1.54 & 901 & & 42-b \\
\hline $\mathrm{CoCr}_{2} \mathrm{Ni}_{3} \mathrm{AlTi}$ & $\begin{array}{c}\text { Two BCC phases }+ \\
\text { FCC }+\mathrm{Al}_{x} \mathrm{Co}\end{array}$ & -102 & 4.3 & 2.23 & 936 & & $42-\mathrm{c}$ \\
\hline $\mathrm{Fe}_{40} \mathrm{Ni}_{20} \mathrm{Co}_{20} \mathrm{Cr}_{20}$ & FCC & 34 & ---- & 4.7 & 862 & $\begin{array}{l}\text { Electrolyte: } 0.1 \\
\text { mol. } \mathrm{L}^{-1} \mathrm{H}_{2} \mathrm{SO}_{4} \\
\text { solution at RT }\end{array}$ & 45 \\
\hline $\mathrm{AlCuCoFeNi}$ & $\mathrm{FCC}+\mathrm{BCC}$ & -58 & 7.9 & ---- & $\begin{array}{ll}--- \\
-1\end{array}$ & \multirow{4}{*}{$\begin{array}{c}\text { Cu-rich FCC } \\
\text { phase; } \mathrm{Al} \text { and } \\
\text { Ni-rich } \mathrm{BCC} \\
\text { phase; Ti and } \\
\text { Cr promoted } \\
\text { grain refinement; } \\
\text { Electrolyte: } 0.5 \\
\text { mol.L } \mathrm{L}^{-1} \mathrm{H}_{2} \mathrm{SO}_{4} \\
\text { solution at RT }\end{array}$} & ${ }^{55}-\mathrm{a}$ \\
\hline $\mathrm{AlCoCuFeNiCr}$ & $\mathrm{BCC}+\mathrm{FCC}$ & -75 & 5.1 & ---- & $\begin{array}{ll}--- \\
\end{array}$ & & ${ }^{55}-\mathrm{b}$ \\
\hline AlCoCuFeNiTi & $\mathrm{FCC}+\mathrm{BCC}+$ Laves & -253 & 44.8 & ---- & ---- & & ${ }^{55}-\mathrm{c}$ \\
\hline AlCoCuFeNiCrTi & $\mathrm{FCC}+\mathrm{BCC}+$ Laves & -256 & 39.6 & ---- & ---- & & ${ }^{55}-\mathrm{d}$ \\
\hline
\end{tabular}


Table 1. Continued...

\begin{tabular}{|c|c|c|c|c|c|c|c|}
\hline Alloy composition & Structure & $\begin{array}{c}\mathrm{E}_{\text {corr }} \\
\left(\mathrm{mV}_{\mathrm{SCE}}\right)\end{array}$ & $\begin{array}{c}\mathrm{i}_{\text {corr }}(\mu \mathrm{A} . \\
\left.\mathrm{cm}^{-2}\right)\end{array}$ & $\begin{array}{c}\mathrm{i}_{\text {pass }}(\mu \mathrm{A} . \\
\left.\mathrm{cm}^{-2}\right)\end{array}$ & $\begin{array}{c}\mathrm{E}_{\mathrm{pit}} \\
\left(\mathrm{mV}_{\mathrm{SCE}}\right) \\
\end{array}$ & $\begin{array}{l}\text { Additional } \\
\text { comments }\end{array}$ & Reference \\
\hline $\mathrm{AlCoCrFeNi}$ & $\begin{array}{c}\mathrm{FCC}+\mathrm{BCC}+\mathrm{B} 2 \\
(\text { ordered } \mathrm{BCC})\end{array}$ & -188 & 30.4 & 2.12 & 1008 & $\begin{array}{c}\text { Annealed at } \\
1100^{\circ} \mathrm{C} \text { for } 48 \mathrm{~h} \\
\text { and quenched in } \\
\text { water; } \mathrm{Cr} \text { enriched } \\
\text { in the } \mathrm{BCC} \text { phase; } \\
\text { Electrolyte: } 0.5 \\
\text { mol. } \mathrm{L}^{-1} \mathrm{H}_{2} \mathrm{SO}_{4} \\
\text { solution at RT }\end{array}$ & 56 \\
\hline $\mathrm{FeCoCrNiAl}_{0.3}$ & FCC & -294 & 17.93 & 2.58 & 901 & $\begin{array}{l}\text { Electrolyte: } 0.5 \\
\text { mol. } \mathrm{L}^{-1} \mathrm{H}_{2} \mathrm{SO}_{4} \\
\text { solution at RT }\end{array}$ & 57 \\
\hline $\mathrm{AlCoCrFeNiTi}_{0.5}$ & ---- & -100 & 0.57 & 26 & 950 & $\begin{array}{l}\text { Electrolyte: } 0.5 \\
\text { mol. } \mathrm{L}^{-1} \mathrm{H}_{2} \mathrm{SO}_{4} \\
\text { solution at RT }\end{array}$ & 58 \\
\hline $\mathrm{CoFeCrNi}$ & FCC & -250 & ---- & 4.63 & ---- & \multirow{4}{*}{ 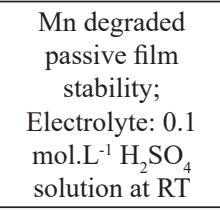 } & ${ }^{59}-\mathrm{a}$ \\
\hline CoFeCrNiMn & FCC & -320 & ---- & 4.37 & ---- & & ${ }^{59}-\mathrm{b}$ \\
\hline$(\mathrm{CoFeNiMn})_{75} \mathrm{Cr}_{25}$ & FCC & -310 & ---- & 4.33 & ---- & & ${ }^{59}-\mathrm{c}$ \\
\hline$(\mathrm{CoFeNi})_{80} \mathrm{Cr}_{20}$ & FCC & -240 & ---- & 3.59 & ---- & & ${ }^{59}-\mathrm{d}$ \\
\hline $\mathrm{CrMnFeNi}$ & FCC & 26 & 191 & ---- & 1568 & \multirow[b]{2}{*}{$\begin{array}{l}\text { Homogeneous } \\
\text { element } \\
\text { distribution in } \\
\text { dendrites and } \\
\text { interdendrites; } \\
\text { Electrolyte: } 0.5 \\
\text { mol. } \mathrm{L}^{-1} \mathrm{H}_{2} \mathrm{SO}_{4} \\
\text { solution at RT }\end{array}$} & ${ }^{60}-\mathrm{a}$ \\
\hline $\mathrm{CrMnFeNiLa}_{0.1}$ & FCC & 73 & 338 & ---- & 1346 & & ${ }^{60}-\mathrm{b}$ \\
\hline
\end{tabular}

Table 2. Alloy composition, structure and corrosion properties of different HEAs. Data obtained in sodium chloride solutions.

\begin{tabular}{|c|c|c|c|c|c|c|c|}
\hline Alloy composition & Structure & $\begin{array}{c}\mathrm{E}_{\text {corr }} \\
\left(\mathrm{mV}_{\mathrm{SCE}}\right)\end{array}$ & $\begin{array}{c}\mathrm{i}_{\text {corr }}(\mu \mathrm{A} . \\
\left.\mathrm{cm}^{-2}\right)\end{array}$ & $\mathrm{i}_{\text {pass }}\left(\mu \mathrm{A} \cdot \mathrm{cm}^{-2}\right)$ & $\mathrm{E}_{\mathrm{pit}}\left(\mathrm{mV}_{\mathrm{SCE}}\right)$ & $\begin{array}{l}\text { Additional } \\
\text { comments }\end{array}$ & Reference \\
\hline $\mathrm{CoCrFeNi}$ & FCC & -234 & ---- & 4.0 & ---- & $\begin{array}{c}\text { Electrolyte: } \\
3.5 \mathrm{wt} \% \% \mathrm{NaCl} \\
\text { solution at } \mathrm{RT}\end{array}$ & 17-a \\
\hline $\mathrm{CoCrFeNiAl}$ & $\begin{array}{c}\mathrm{BCC}+\mathrm{B} 2 \\
\text { (ordered } \mathrm{BCC} \text { ) }\end{array}$ & -300 & ---- & 6.3 & 667 & & \\
\hline $\mathrm{Al}_{0.3} \mathrm{CoCrFeNi}$ & FCC & -195 & 0.0835 & 1.24 & 460 & $\begin{array}{c}\text { Increasing Al } \\
\text { content reduces } \\
\text { the corrosion } \\
\text { resistance (thicker, } \\
\text { less stable passive } \\
\text { film); Electrolyte: } \\
3.5 \text { wt. } \% \mathrm{NaCl} \\
\text { solution at RT }\end{array}$ & 27 \\
\hline $\mathrm{CoCrFeNiMo}_{0.4}$ & $\begin{array}{c}\mathrm{FCC}+\mathrm{Cr} \text { and } \\
\text { Mo-rich sigma } \\
\text { phase }\end{array}$ & -261 & 0.082 & 16 & 948 & $\begin{array}{c}\text { Electrolyte: } \\
3.5 \text { wt. } \% \mathrm{NaCl} \\
\text { solution at RT }\end{array}$ & 30 \\
\hline $\mathrm{FeCoNiCr}_{0.5}$ & FCC & -319 & 2.36 & ---- & 453 & $\begin{array}{c}\text { Electrolyte: } \\
3.5 \text { wt. } \% \mathrm{NaCl} \\
\text { solution at RT }\end{array}$ & 31 \\
\hline $\mathrm{AlCr}_{2.5} \mathrm{FeNi}_{2} \mathrm{Cu}_{1.6}$ & $\mathrm{FCC}+\mathrm{BCC}$ & -516 & 1.25 & --- & -21 & $\begin{array}{c}\text { Electrolyte: } \\
3.5 \text { wt. } \% \mathrm{NaCl} \\
\text { solution at } \mathrm{RT}\end{array}$ & 34 \\
\hline $\mathrm{CoCrFeMnNi}$ & FCC & -230 & 0.14 & ---- & ---- & $\begin{array}{c}\text { Electrolyte: } \\
3.5 \text { wt. } \% \mathrm{NaCl} \\
\text { solution at } \mathrm{RT}\end{array}$ & 37 \\
\hline
\end{tabular}


Table 2. Continued...

\begin{tabular}{|c|c|c|c|c|c|c|c|}
\hline Alloy composition & Structure & $\begin{array}{c}\mathrm{E}_{\text {corr }} \\
\left(\mathrm{mV}_{\mathrm{SCE}}\right)\end{array}$ & $\begin{array}{c}\mathrm{i}_{\text {corr }}(\mu \mathrm{A} . \\
\left.\mathrm{cm}^{-2}\right)\end{array}$ & $\mathrm{i}_{\text {pass }}\left(\mu \mathrm{A} \cdot \mathrm{cm}^{-2}\right)$ & $\mathrm{E}_{\mathrm{pit}}\left(\mathrm{mV}_{\mathrm{SCE}}\right)$ & $\begin{array}{l}\text { Additional } \\
\text { comments }\end{array}$ & Reference \\
\hline $\mathrm{Al}_{2} \mathrm{CrFeCoCuTiNi}$ & $\mathrm{FCC}+\mathrm{BCC}$ & -220 & 13 & ---- & ---- & $\begin{array}{l}\text { Increasing } \mathrm{Ni} \\
\text { contents reduced } \\
\text { the corrosion } \\
\text { resistance; } \\
\text { Electrolyte: } \\
3.5 \text { wt. } \% \mathrm{NaCl} \\
\text { solution at } \mathrm{RT}\end{array}$ & 40-a \\
\hline $\mathrm{Al}_{2} \mathrm{CrFeCoCuTiNi}_{2}$ & $\mathrm{FCC}+\mathrm{BCC}$ & -500 & 67 & ---- & ---- & & 40-b \\
\hline $\mathrm{Al}_{2} \mathrm{CrFeCo}_{10} \mathrm{CuNiTi}$ & ---- & -220 & 0.013 & ---- & & Laser-cladded & $\begin{array}{ll}3-\mathrm{a} \\
\end{array}$ \\
\hline $\mathrm{Al}_{2} \mathrm{CrFeCo}_{1.5} \mathrm{CuNiTi}$ & ---- & -510 & 0.074 & ---- & $\begin{array}{l}\text { No passivity } \\
\text { breakdown up to } \\
1.2 \mathrm{~V}_{\mathrm{SCE}}\end{array}$ & $\begin{array}{l}\text { coatings on Q235 } \\
\text { steel substrate; } \\
\text { Electrolyte: } \\
3.5 \text { wt.\% } \mathrm{NaCl} \\
\text { solution at RT }\end{array}$ & 43-b \\
\hline $\mathrm{Co}_{2} \mathrm{CrCuFeMnNi}$ & $\begin{array}{l}\text { Two FCC } \\
\text { phases }\end{array}$ & -787 & 6.95 & ---- & 19 & $\begin{array}{l}\text { Sintered alloy; the } \\
\text { FCC phase with } \\
\text { higher Co content } \\
\text { is more resistant } \\
\text { to corrosion; } \\
\text { Electrolyte: } \\
3.5 \text { wt. } \% \mathrm{NaCl} \\
\text { solution at RT }\end{array}$ & 44 \\
\hline CrMnFeCoNi & $\mathrm{FCC}$ & 142.5 & 0.105 & ---- & 460 & $\begin{array}{l}\text { Cr-depleted } \\
\text { interdendrites are } \\
\text { the starting point } \\
\text { for corrosion; } \\
\text { Electrolyte: } \\
3.5 \text { wt. } \% \mathrm{NaCl} \\
\text { solution at RT }\end{array}$ & 61 \\
\hline ' $(\mathrm{CoCrFeNi})_{95} \mathrm{Nb}_{5}$ & $\mathrm{FCC}$ & -128 & 7.23 & 41.3 & 502 & $\begin{array}{c}\text { Plasma sprayed } \\
\text { coating on Q235 } \\
\text { steel substrate; } \\
\text { Electrolyte: } \\
3.5 \text { wt. } \% \mathrm{NaCl} \\
\text { solution at RT }\end{array}$ & 62 \\
\hline $\mathrm{Al}_{0.9} \mathrm{CoCrFeNi}$ & $\begin{array}{c}\mathrm{FCC}+\mathrm{BCC} \\
+\mathrm{B} 2 \text { (ordered } \\
\mathrm{BCC})\end{array}$ & -216 & 0.093 & ---- & 164 & $\begin{array}{c}\text { Electrolyte: } \\
3.5 \mathrm{wt} . \% \mathrm{NaCl} \\
\text { solution at RT }\end{array}$ & 63-a \\
\hline $\mathrm{Al}_{0.9} \mathrm{CoCrFeNiTi}_{0.5}$ & $\begin{array}{c}\mathrm{BCC}+\mathrm{B} 2 \\
\text { (ordered } \mathrm{BCC} \text { ) } \\
+\mathrm{Fe}-\mathrm{Cr} \text { sigma } \\
\text { phase }\end{array}$ & -347 & 0.310 & ---- & 184 & & 63-b \\
\hline $\mathrm{CoCrFeNi}_{2}$ & FCC & -290 & 0.129 & ---- & 320 & $\begin{array}{l}\text { Mo enrichment } \\
\text { in the passive } \\
\text { film reduces } \\
\text { pitting corrosion } \\
\text { susceptibility; } \\
\text { Electrolyte: } \\
3.5 \text { wt. } \% \mathrm{NaCl} \\
\text { solution at RT }\end{array}$ & 64-a \\
\hline $\mathrm{CoCrFeNi}_{2} \mathrm{Mo}_{0.25}$ & $\mathrm{FCC}+\mathrm{HCP}$ & -260 & 0.125 & ---- & 910 & & 64-b \\
\hline$(\mathrm{Fe}-\mathrm{Co}-\mathrm{Ni})_{15}(\mathrm{Al}-\mathrm{Ti}-\mathrm{Zr})_{85}$ & $\begin{array}{c}\text { FCC }+ \\
\text { amorphous } \\
\text { phase }\end{array}$ & -460 & 2.046 & ---- & ---- & $\begin{array}{l}\text { Multi-component } \\
\text { films obtained } \\
\text { by sputtering } \\
\text { on Si substrate; } \\
\text { Electrolyte: } \\
3.5 \text { wt.\% } \% \mathrm{NaCl} \\
\text { solution at RT }\end{array}$ & 65-a \\
\hline$(\mathrm{Fe}-\mathrm{Co}-\mathrm{Ni})_{20}(\mathrm{Al}-\mathrm{Ti}-\mathrm{Zr})_{80}$ & $\begin{array}{c}\mathrm{FCC}+ \\
\text { amorphous } \\
\text { phase }\end{array}$ & -281 & 0.586 & ---- & ---- & & 65-b \\
\hline
\end{tabular}


Table 2. Continued...

\begin{tabular}{|c|c|c|c|c|c|c|c|}
\hline Alloy composition & Structure & $\underset{\left(\mathrm{mV}_{\mathrm{SCE}}\right)}{\mathrm{E}_{\text {corr }}}$ & $\begin{array}{c}\mathrm{i}_{\text {corr }}(\mu \mathrm{A} . \\
\left.\mathrm{cm}^{-2}\right)\end{array}$ & $\mathrm{i}_{\text {pass }}\left(\mu \mathrm{A} \cdot \mathrm{cm}^{-2}\right)$ & $\mathrm{E}_{\mathrm{pit}}\left(\mathrm{mV}_{\mathrm{SCE}}\right)$ & $\begin{array}{l}\text { Additional } \\
\text { comments }\end{array}$ & Reference \\
\hline$(\mathrm{Fe}-\mathrm{Co}-\mathrm{Ni})_{25}(\mathrm{Al}-\mathrm{Ti}-\mathrm{Zr})_{75}$ & $\begin{array}{c}\text { FCC }+ \\
\text { amorphous } \\
\text { phase }\end{array}$ & -260 & 0.062 & --- & --- & & $65-\mathrm{c}$ \\
\hline $\mathrm{Al}_{0.3} \mathrm{Cr}_{0.5} \mathrm{FeCoNi}$ & FCC & -185 & 0.1 & ---- & 350 & $\begin{array}{l}\text { Increasing the } \\
\text { Cr content in } \\
\text { the alloy, the } \\
\text { FCC and BCC } \\
\text { phases became } \\
\text { enriched with } \\
\text { Cr; Electrolyte: } \\
3.5 \text { wt. } \% \text { NaCl } \\
\text { solution at RT }\end{array}$ & 66-a \\
\hline
\end{tabular}

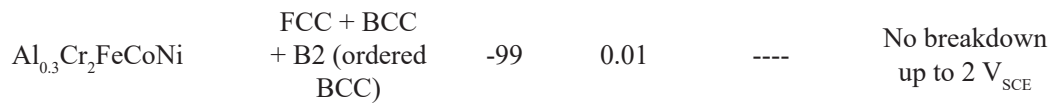

\begin{tabular}{|c|c|c|c|c|c|c|c|}
\hline $\mathrm{TiCrVNb}_{0.5} \mathrm{Al}_{0.5}$ & $\mathrm{BCC}$ & -450 & 0.089 & ---- & 1950 & $\begin{array}{c}\text { Uniform } \\
\text { distribution of } \\
\text { elements without } \\
\text { segregation; } \\
\text { Electrolyte: } \\
3.5 \text { wt. } \% \mathrm{NaCl} \\
\text { solution at } \mathrm{RT} \\
\end{array}$ & 67 \\
\hline $\mathrm{Al}_{0.5} \mathrm{CoCrFeNi}$ & $\mathrm{FCC}$ & -570 & 0.17 & ---- & 130 & $\begin{array}{c}\text { As-cast condition; } \\
\text { Electrolyte: } \\
3.5 \mathrm{wt} \% \mathrm{NaCl} \\
\text { solution at } \mathrm{RT}\end{array}$ & 68 \\
\hline $\mathrm{CoCrFeNiMo}_{0.2}$ & $\mathrm{FCC}$ & -192 & 0.00184 & ---- & 827 & $\begin{array}{c}\text { Mo enhances } \\
\text { passivity (passive } \\
\text { film composition } \\
\text { not shown); } \\
\text { Electrolyte: } \\
3.5 \text { wt. } \% \mathrm{NaCl} \\
\text { solution at RT }\end{array}$ & 69 \\
\hline$(\mathrm{CoCrFeNi})_{98} \mathrm{Mo}_{2}$ & $\mathrm{FCC}$ & -191 & 0.07 & ---- & ---- & $\begin{array}{l}\text { Aging at } 900 \\
{ }^{\circ} \mathrm{C} \text { for } 16 \mathrm{~h}, \\
\text { followed by water } \\
\text { quenching; Co and } \\
\text { Fe-rich dendrites; } \\
\mathrm{Cr} \text { and Mo-rich } \\
\text { interdendrites; } \\
\text { Electrolyte: } \\
3.5 \text { wt. } \% \mathrm{NaCl} \\
\text { solution at } \mathrm{RT}\end{array}$ & 70-a \\
\hline$(\mathrm{CoCrFeNi})_{98} \mathrm{Mo}_{3}$ & $\begin{array}{c}\text { FCC + } \\
\text { Mo-rich } \\
\text { precipitates }\end{array}$ & -194 & 0.22 & ---- & ---- & & $70-\mathrm{b}$ \\
\hline $\mathrm{AlCoCrFeNiCu}_{0.5}$ & $\mathrm{FCC}+\mathrm{BCC}$ & -282 & 1.77 & --- & ---- & $\begin{array}{l}\text { Equiaxed grains } \\
\text { (grain size smaller } \\
\text { than } 30 \mu \mathrm{m} \text { ); no } \\
\mathrm{Cu} \text { segregation in } \\
\text { grain boundaries; } \\
\text { Electrolyte: } \\
\text { artificial seawater }\end{array}$ & 71 \\
\hline
\end{tabular}


Table 2. Continued...

\begin{tabular}{|c|c|c|c|c|c|c|c|}
\hline Alloy composition & Structure & $\begin{array}{c}\mathrm{E}_{\text {corr }} \\
\left(\mathrm{mV}_{\mathrm{SCE}}\right)\end{array}$ & $\begin{array}{c}\mathrm{i}_{\text {corr }}(\mu \mathrm{A} . \\
\left.\mathrm{cm}^{-2}\right)\end{array}$ & $\mathrm{i}_{\text {pass }}\left(\mu \mathrm{A} \cdot \mathrm{cm}^{-2}\right)$ & $\mathrm{E}_{\mathrm{pit}}\left(\mathrm{mV}_{\mathrm{SCE}}\right)$ & $\begin{array}{l}\text { Additional } \\
\text { comments }\end{array}$ & Reference \\
\hline $\mathrm{CoCrFeNi}$ & FCC & -238 & ---- & ---- & 575 & $\begin{array}{l}\text { Sn increases } \\
\text { the corrosion } \\
\text { resistance of } \\
\text { CoCrFeNi, } \\
\text { whereas } \mathrm{Al} \text { and } \\
\text { Cu decrease; } \\
\text { Electrolyte: } \\
\text { 3.5 wt. } \% \mathrm{NaCl} \\
\text { solution at } \mathrm{RT}\end{array}$ & 72-a \\
\hline CoCrFeNiAl & $\begin{array}{c}\mathrm{BCC}+\mathrm{B} 2 \\
\text { (ordered BCC) }\end{array}$ & -303 & ---- & ---- & 316 & & 72-b \\
\hline $\mathrm{CoCrFeNiCu}$ & $\begin{array}{c}\text { FCC (matrix) } \\
+\mathrm{Cu}-\mathrm{rich} \\
\mathrm{FCC}(\mathrm{Cu} \\
\text { segregation })\end{array}$ & -161 & ---- & ---- & -50 & & $72-\mathrm{c}$ \\
\hline CoCrFeNiSn & $\mathrm{FCC}+\mathrm{HCP}$ & -233 & $\begin{array}{c}--- \\
\end{array}$ & ---- & 1118 & & $72-\mathrm{d}$ \\
\hline $\mathrm{CoFeMn} n_{1.2} \mathrm{NiGa}_{0.8}$ & $\mathrm{FCC}+\mathrm{BCC}$ & -416 & 1.11 & ---- & 790 & $\begin{array}{c}\text { Electrolyte: } \\
3.5 \mathrm{wt} \% \mathrm{NaCl} \\
\text { solution at } \mathrm{RT}\end{array}$ & 73 \\
\hline $\mathrm{Al}_{10} \mathrm{Co}_{24} \mathrm{Cr}_{10} \mathrm{Fe}_{15} \mathrm{Ni}_{34} \mathrm{Ti}_{6} \mathrm{Cu}_{1}$ & FCC & -63 & 0.022 & ---- & ---- & $\begin{array}{c}\mathrm{Fe}, \mathrm{Co} \text { and } \mathrm{Cr} \\
\text { enriched in the } \\
\text { dendrite region; } \mathrm{Al} \\
\text { and Ti enriched in } \\
\text { the interdendrite } \\
\text { region; } \\
\text { Electrolyte: } \\
3.5 \text { wt.\% } \mathrm{NaCl} \\
\text { solution at } \mathrm{RT}\end{array}$ & 74 \\
\hline $\mathrm{CoCrFeNiGe}_{0.3}$ & FCC & -34 & 0.56 & 2.51 & 1410 & $\begin{array}{l}\mathrm{GeO}_{2} \text { oxide } \\
\text { present in the } \\
\text { passive film } \\
\text { increases the } \\
\text { corrosion } \\
\text { resistance } \\
\text { with respect to } \\
\text { CoCrFeNi HEA; } \\
\text { Electrolyte: } \\
3.5 \text { wt.\% } \mathrm{NaCl} \\
\text { solution at RT }\end{array}$ & 75 \\
\hline $\mathrm{Co}_{30} \mathrm{Cr}_{20} \mathrm{Fe}_{30} \mathrm{Ni}_{20}$ & ---- & 12 & 2.32 & 2.01 & 970 & $\begin{array}{c}\text { Electrolyte: } \\
3.5 \mathrm{wt} \% \mathrm{NaCl} \\
\text { solution at } \mathrm{RT}\end{array}$ & 76 \\
\hline $\mathrm{Ni}_{2} \mathrm{CrFeMo}_{02}$ & ---- & -179 & 0.896 & 5.404 & 920 & & 77 \\
\hline
\end{tabular}

composition is mentioned to allow comparing the alloys in compatible environments. Additional comments are also given to highlight critical aspects related to distribution of elements, as well as the effect of specific alloying elements on the corrosion resistance and passive film stability of the HEAs. Also, the heat treatment condition of the alloy is mentioned, whenever it is a relevant part of the experimental procedure. If not mentioned, the alloy was not heat treated after processing. The information shown in these tables will serve as the basis for the materials selection procedure developed in the next section.

\section{Materials Selection}

The first step of the materials selection process based on the Ashby method is translation. In this step, design requirements are defined such as the function played by the material, the objective (what is to be maximized), constraints and free variables. A general function will be defined for this purpose, considering a structural component manufactured from a multi-principal alloy. The objective is to maximize the corrosion resistance of the alloy. The constraints are related to the corrosive environment (acidic or sodium chloride solutions). The free variables are the choice of material, component size and section area. This information is shown in Table 3.

The second step is to screen the candidates based on the constraints. This part of the selection process can be promptly done based on the information shown in Tables 1 and 2, as the corrosion properties displayed therein were obtained for acidic and sodium chloride solutions, respectively. However, 
from this point on, we will separate our selection process in two parts. The first one is devoted to materials to be used in acidic environments and the second one is referred to sodium chloride solutions. Such assumption makes the outputs of the materials selection procedure more accurate, as we avoid comparing materials exposed to different environments that could lead to distinct corrosion behaviors.

By defining the constraints, the candidates that should be ranked in the third step are taken from Tables 1 and 2 . Ranking is based on the objective previously defined in Table 3. Now, the central question is what criterion should be considered to maximize the corrosion resistance of the HEAs. A good measure of the uniform corrosion susceptibility can be based on the corrosion current density $\left(i_{\text {cor }}\right)$, as a criterion to rank the anodic dissolution rate of different materials. In acidic solutions, graphical representation of $i_{\text {corr }}$ and $\mathrm{E}_{\text {corr }}$ can provide useful insights on the uniform corrosion resistance of $\mathrm{HEAs}^{13}$. Low values of $\mathrm{i}_{\text {corr }}$ indicate the best corrosion resistance with respect to slow corrosion kinetics.

However, for passive metals in sodium chloride solutions, as many types of HEAs, $i_{\text {corr }}$ is not the most suitable parameter, as localized corrosion is the dominant degradation mode. In this case, pitting potential $\left(\mathrm{E}_{\mathrm{pit}}\right)$ and the passive range $\left(\mathrm{E}_{\text {pit }}-\mathrm{E}_{\text {corr }}\right)$. Large values of both parameters indicate the best resistance to localized corrosion.

\subsection{Acidic solutions}

Firstly, the corrosion resistance of HEAs in acidic electrolytes is considered. In Figure 1, the alloys whose corrosion properties are shown in Table 1 are displayed in a materials property chart of $\mathrm{E}_{\text {corr }}$ vs. $\mathrm{i}_{\text {corr }}$. Each bubble represents a different alloy. The numbers in square brackets are referred to the references from which the corrosion properties were collected. Data for a 304 stainless steel, $\mathrm{Cu}$, Ti and Ni alloys are also shown in this chart to allow for the comparison of its corrosion resistance with that of the HEAs. To avoid misleading conclusions, only alloys immersed in the same type of electrolyte were plotted in Figure 1 ( 0.5 mol.L-1 $\mathrm{H}_{2} \mathrm{SO}_{4}$ solution at room temperature). Hence, alloys from references $\left[{ }^{45}\right]$ and $\left[{ }^{59}\right]$, which were immersed in a more diluted solution $\left(0.5\right.$ mol. $\left.\mathrm{L}^{-1} \mathrm{H}_{2} \mathrm{SO}_{4}\right)$, were not included in Figure 1.

Based on the design criterion defined in the beginning of the present section, the most corrosion resistant materials are those with lowest values of $i_{\text {corr }}$. They are indicated by the green and yellow balloons in Figure 1. The best candidates are ranked in the following order: ${ }^{5}>{ }^{42}-\mathrm{b}>{ }^{30}>{ }^{42}-\mathrm{c}>{ }^{55}-\mathrm{b}$ $>{ }^{42}-\mathrm{a}>{ }^{55}$-a.

As shown in Table $1^{58}$, is the AlCoCrFeNiTi ${ }_{0.5}$ alloy, ${ }^{42}-\mathrm{b}$ is the $\mathrm{Co}_{2} \mathrm{Cr}_{2} \mathrm{Ni}_{2} \mathrm{AlTi}$ alloy, and ${ }^{30}$ is the $\mathrm{CoCrFeNiMo}{ }_{0.4}$ alloy. These alloys are the top ranked materials in Figure 1, followed by the Ti-6Al-4V alloy ${ }^{79}$. It is evident that the corrosion resistance of HEAs suppresses that of conventional $\mathrm{Cu}$ and Ni alloys, 304 stainless steel, and even of the Ti-6Al-4V alloy, depending on the alloy composition. Zhang et al. ${ }^{58}$ did not evaluate the composition of the passive film. They associated the high corrosion resistance of the $\mathrm{AlCoCrFeNiTi}_{0.5}$ alloy with an increase in the proportion of the Al-rich BCC phase with respect to the FCC matrix as the $\mathrm{Al}$ content increased in the alloy. With regard to the good performance of $\mathrm{Co}_{2} \mathrm{Cr}_{2} \mathrm{Ni}_{2} \mathrm{AlTi}$ alloy against corrosion, Huang et al. ${ }^{42}$ explain its high corrosion resistance based on the stability of the passive film that is promoted by the concomitant presence of $\mathrm{Co}$ and $\mathrm{Cr}$. According to the authors, Co potentially inhibits $\mathrm{Cr}_{2} \mathrm{O}_{3}$ hydrolysis in the sulfuric acid solution, thus promoting the stability of the passive film. In fact, if one considers the pitting potential for this alloy (Table 1), it is higher than $900 \mathrm{mV}_{\mathrm{SCE}}$, denoting the formation of a stable surface oxide film. Another effect mentioned by the authors is that Co suppresses diffusion of sulfur, further enhancing the passive film stability by avoiding the formation of NiS. The results shown in Figure 1 give a clear indication that combining $\mathrm{Co}$ and $\mathrm{Cr}$ is an effective method of enhancing the corrosion resistance of HEAs in the CoCrNiAlTi system, provided that $\mathrm{Ni}$ is not excessively increased, as it would favor the formation of a more porous and non-protective passive film. Shang et al. ${ }^{30}$ ascribed the high corrosion resistance of $\mathrm{CoCrFeNiMo}{ }_{0.4}$ alloy to the beneficial effect of Mo when present in the $\mathrm{Mo}^{6+}$ state $\left(\mathrm{MoO}_{3}\right)$, provided it does not lead to the formation of $\sigma$-phase that may originate galvanic coupling with surrounding Mo-depleted regions, accelerating the corrosion rate. Hence, the passive film composition is critical for achieving stable surfaces. This is in line with the study by Scully et al. ${ }^{54}$, which highlighted the importance of the passive film to obtain corrosion-resistant HEAs. However, other aspects that must be taken into account to develop corrosion resistant HEAs, as also highlighted by Scully et al., such as phase stability, substrate properties (metal-metal bonds), and structural heterogeneities.

The candidates with intermediate performance are circled by the yellow balloon in Figure 1. These alloys are described as follows from Table $1:{ }^{42}$-c is the $\mathrm{CoCr}_{2} \mathrm{Ni}_{3} \mathrm{AlTi}$ alloy, ${ }^{55}$-b is the $\mathrm{AlCoCuFeNiCr}$ alloy, ${ }^{42}$-a is the $\mathrm{CoCr}_{2} \mathrm{Ni}_{2} \mathrm{AlTi}$ alloy, and ${ }^{55}$-a is the $\mathrm{AlCuCoFeNi}$ alloy. As mentioned in the previous paragraph, Huang et al. ${ }^{42}$ explain the different corrosion resistances of the CoCrNiAlTi HEAs based on the composition of the passive film, depending on the $\mathrm{Cr}_{2} \mathrm{O}_{3}$ content. For example, the reason why ${ }^{42}$-c displays larger $i_{\text {corr }}$ than ${ }^{42}-b$ is attributed to the formation of the unstable NiS layer due to its relatively high Ni content. Passive film stability is also the key to explain the relatively low $i_{\text {corr }}$ of ${ }^{42}$-a with respect to ${ }^{42}$-c. In this case, the low Co content of ${ }^{42}$-a enables the formation of a less stable passive film when compared to ${ }^{42}-b$. The beneficial role of $\mathrm{Cr}$ addition in enabling the formation

Table 3. Design requirements for corrosion resistant HEAs.

\begin{tabular}{cc}
\hline & Design requirements \\
\hline Function & Structural component \\
\hline Constraints & Acidic or sodium chloride electrolytes \\
\hline Objectives & To maximize the corrosion resistance \\
\hline Free variables & Component size and section area; choice of material \\
\hline
\end{tabular}




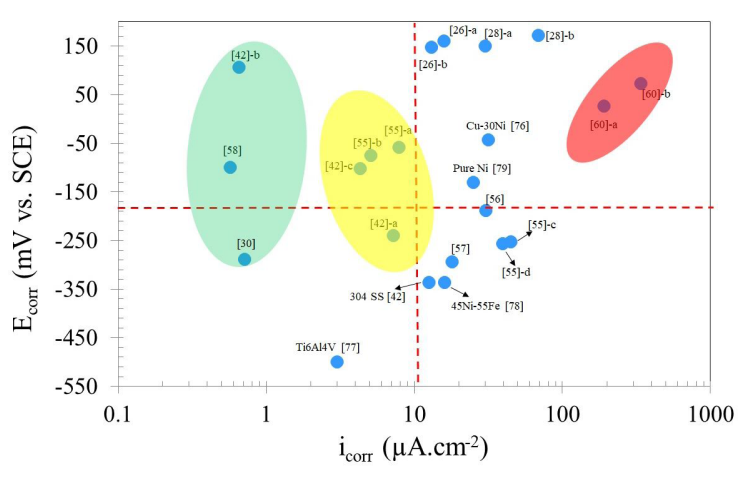

Figure 1. Ashby chart showing $\mathrm{E}_{\text {corr }}$ vs. $\mathrm{i}_{\text {corr }}$ for the alloys displayed in Table 1 (corrosion properties in acidic solution).

of a stable passive film is also mentioned by Xiao et al. ${ }^{55}$ to explain the low $i_{\text {corr }}$ of ${ }^{55}-b$ when compared to ${ }^{55}$-a.

Manganese is often reported as a deleterious element with respect to the passive film stability of $\mathrm{HEAs}^{46,47}$. In fact, the candidates with the worst corrosion resistance in Figure 1 are ${ }^{60}$-a and ${ }^{60}-b$, as indicated by the red balloon. As shown in Table 1, Mn is a common alloying element for these alloys. There are, of course, further aspects that must be taken into account to explain the corrosion resistance of Mn-containing HEAs. The simple presence of Mn is not sufficient, per se, to hamper the corrosion resistance of the alloy. Interaction with other alloying elements is important, especially with regard to the composition of the passive film. However, it is known that manganese oxides are not as protective as other oxides, such as $\mathrm{Cr}_{2} \mathrm{O}_{3}$. Hence, alloy composition must be carefully designed to enhance passive film stability.

To further document the results displayed in the Ashby chart in Figure 1, additional information were collected from the references shown in Table 1. In this respect, the values of passive current densities $\left(i_{\text {pass }}\right)$ of selected HEAs are plotted in Figure 2. Only the alloys for which the $i_{\text {pass }}$ values were given in the corresponding reference have been included in Figure 2. This parameter can be envisaged as a measure of the anodic dissolution rate for passive metals. Low values of $i_{\text {pass }}$ indicate a slow dissolution rate. Although it is not always reported, it is useful to complement the insightful information plotted in the $\mathrm{E}_{\text {corr }} \mathrm{x}_{\text {corr }}$ Ashby chart. The data

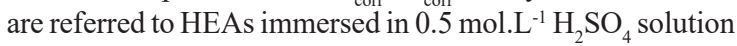
at room temperature, as in Figure 1.

If one compares the information in the bar chart shown in Figure 1 with the Ashby chart shown in Figure 1, some noticeable differences are seen. The most striking one is related to $\mathrm{AlCoCrFeNiTi}_{0.5}$ alloy ${ }^{58}$. It displays the lowest value of $i_{\text {corr }}$ but, as shown in Figure 2, its $i_{\text {pass }}$ is the highest one, indicating it is not a good candidate if one takes the resistance to localized corrosion into account. Similarly, the $\mathrm{CoCrFeNiMo}_{0.4}$ alloy $^{30}$, which is the third best candidate according to the $i_{\text {corr }}$ values shown in Figure 1, is only at an intermediate position in Figure 2. Conversely, the best candidates in Figure 2 are in the following sequence: ${ }^{42}-b>56>{ }^{42}-c>42$-a. So, alloys of the CoCrNiAlTi system ${ }^{42}$ have a remarkable corrosion resistance, independently if one looks to the general corrosion $\left(\mathrm{i}_{\text {corr }}\right)$ or localized corrosion resistance $\left(\mathrm{i}_{\text {pass }}\right)$. This result confirms the crucial role of passive film composition linked to the alloy design to yield the formation of a stable material against corrosion. If the HEA displays passivity, it is imperative to evaluate the electrochemical parameters related to localized corrosion in order to have a clear picture of the corrosion resistance in acidic solutions.

\section{2. $\mathrm{NaCl}$ solution}

The selection criteria for alloys immersed in sodium chloride solutions will follow a different approach from that discussed in the previous section. In this case, passivity breakdown is a major concern, as many HEA systems behave as passive metals in chloride-containing solutions. Consequently, pitting corrosion parameters should be of prime interest to select the most corrosion resistant materials instead of uniform corrosion parameters (corrosion potential and corrosion current density). In this regard, the Ashby chart displayed in Figure 3 is plotted, showing pit potentials $\left(\mathrm{E}_{\mathrm{pit}}\right)$ as a function of the passive range $\left(\mathrm{E}_{\mathrm{pit}}-\mathrm{E}_{\mathrm{corr}}\right)$, based on the data presented in Table 2. The conventional 304 stainless steel, Ti-6Al-4V $\mathrm{V}^{82}$ and Ti-6Al-3Nb-2Zr-1 $\mathrm{Mo}^{83}$ alloys are also plotted as references. The most corrosion resistant materials should present large values of both parameters, indicating the stability of the passive film in the sodium chloride solution. The passive current density $\left(\mathrm{i}_{\text {pass }}\right)$ could also be used as a design criterion for selecting corrosion resistant alloys in sodium chloride solutions, as it is a measure of the anodic dissolution rate for passive metals. However, as it is only available for a minor part of the alloys displayed in Table 2, it was not included in this chart. All alloys displayed in Figure 3 were immersed in 3.5 wt. $\% \mathrm{NaCl}$ solution at room temperature, allowing an accurate comparison of their corrosion parameters. As in Figure 1, each bubble represents a different alloy, and the numbers in square brackets are the references from which the data were collected.

Most alloys are concentrated in the third quadrant of the chart, as shown in Figure 3. There are three alloys in the first quadrant that show superior resistance to localized corrosion, allying large $\mathrm{E}_{\mathrm{pit}}$ and passive range values, along with the Ti-6Al-3Nb-2Zr-1Mo ${ }^{83}$ alloy. Now, to clearly rank the candidates it is necessary to make additional assumptions. The best choice would be the one that gives the best compromise between the two parameters shown in the Ashby chart. It is evident from Figure 3 that ${ }^{66}-\mathrm{b}$ and ${ }^{67}$ are the best candidates, followed by ${ }^{75}$. However, the choice between ${ }^{66}-\mathrm{b}$ and ${ }^{67}$ is not clear at this point. To make it more evident, it is mandatory to give a further step to optimize the selection process. It is imperative to seek for the best compromise between the two properties. There are different ways of managing this dilemma. One simple solution is to ascribe different weights to each parameter, thus giving more importance to one of them in the selection process. Although this is an arbitrary decision, it is very useful, if employed with a fair assessment of the available information on the material's performance to the intended application. A more quantitative approach can be derived from the Ashby method based on conflicting objectives, and the use of penalty functions. The interested reader can find detailed information about this method in reference ${ }^{25}$. Briefly, a penalty function ( $Z$ ) can be expressed as displayed in Equation 1, where $\alpha$ is called exchange constant. It is a 
measure of the increase in penalty when a unit increment of a specific performance metric (P), if all other metrics are constant. $\mathrm{P}$ is a performance metric. Each objective of the materials selection process is a performance metric ${ }^{25}$.

$$
Z=a_{1} \cdot P_{1}+a_{2} P_{2}+a_{3} P_{3} \ldots
$$

In this work, we adopt the second strategy in order to more clearly view the distinct performances of the alloys in Figure 3. Following this approach, the data in Figure 3 were replotted in the trade-off plot shown in Figure 4, displaying the reciprocal of the metric parameters $\left(1 / \mathrm{E}_{\mathrm{pit}}\right.$ and $1 /\left(\mathrm{E}_{\mathrm{pit}}-\mathrm{E}_{\text {corr }}\right)$ in the plot axes. This step is needed because, when penalty functions are used, the best choice is the one that minimizes its value ${ }^{25}$. Thus, to select the most corrosion resistant HEAs we need to choose the candidates with the lowest values of $1 / \mathrm{E}_{\text {pit }}$ and $1 /\left(\mathrm{E}_{\text {pit }}-\mathrm{E}_{\text {corr }}\right)$ in Figure 4 . We can, therefore, define a penalty function $(Z)$, as shown in Equation 2, where $\alpha_{1}$ and $\alpha_{2}$ are exchange constants whose values are arbitrary, depending on the relative weights given by the designer to each performance metrics. In the present work, we will consider the same weight to both metrics $\left(\mathrm{E}_{\mathrm{pit}}\right.$ and $\left.\mathrm{E}_{\mathrm{pit}}-\mathrm{E}_{\text {corr }}\right)$, so $\alpha_{1}$ is equal to $\alpha_{2}$.

$$
Z=a_{1} .1 / E_{p i t}+a_{2} .1 /\left(E_{p i t}-E_{c o r r}\right)
$$

The trade-off surface gives a visual indication of the best candidates. They are located close to the surface, giving

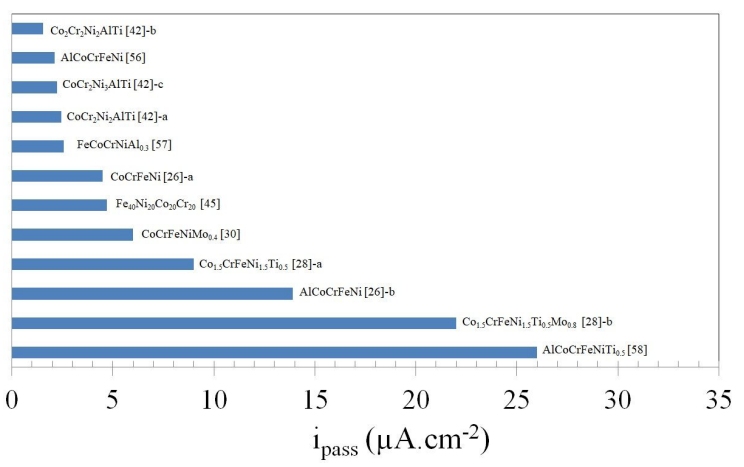

Figure 2. Bar chart showing the values of $\mathrm{i}_{\text {pass }}$ for selected HEAs in 0.5 mol. $\mathrm{L}^{-1} \mathrm{H}_{2} \mathrm{SO}_{4}$ solution at room temperature.

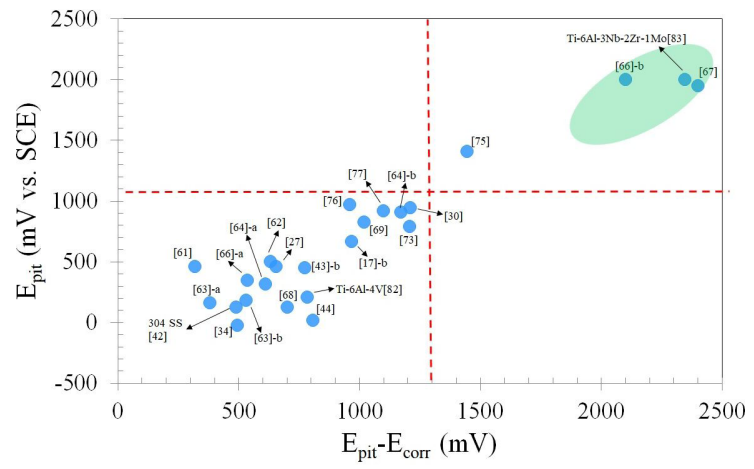

Figure 3. Ashby chart showing $\mathrm{E}_{\mathrm{pit}}$ vs. $\mathrm{E}_{\mathrm{pit}}-\mathrm{E}_{\text {corr }}$ for the alloys displayed in Table 2 (corrosion properties in sodium chloride solution). the lowest values of $\mathrm{Z}$. It is evident that the best candidates are $^{67}$ and $66-b$, followed by ${ }^{75}$. However, the candidate that gives the best compromise between the two performance metrics is ${ }^{67}$, as it minimizes the value of the penalty function. As shown in Table 2, ${ }^{67}$ is the $\mathrm{TiCrVNb}_{0.5} \mathrm{Al}_{0.5}$ alloy, ${ }^{66}-\mathrm{b}$ is the $\mathrm{Al}_{0.3} \mathrm{Cr}_{2} \mathrm{FeCoNi}$ alloy and ${ }^{75}$ is the $\mathrm{CoCrFeNiGe}{ }_{0.3}$ alloy. The best candidate is the $\mathrm{TiCrVNb}{ }_{0.5} \mathrm{Al}_{0.5}$ alloy manufactured by $\mathrm{Li}$ et al. ${ }^{67}$. It is a single phase BCC alloy. According to the authors, this fact, allied to the presence of passivating elements such as $\mathrm{Ti}, \mathrm{Nb}$ and $\mathrm{Cr}$ in the passive film, promoted the formation of a highly stable passive film. The main components in the passive film were the highly stable species $\mathrm{TiO}_{2}$ and $\mathrm{Cr}_{2} \mathrm{O}_{3}$, thus giving rise to a remarkable resistance against pitting corrosion. The second best candidate is the $\mathrm{Al}_{03} \mathrm{Cr}_{2.0} \mathrm{FeCoNi}$ alloy developed by Yan et al. ${ }^{66}$. Chromium addition to the $\mathrm{Al}_{0.3} \mathrm{FeCoNi}$ alloy enabled the enrichment of $\mathrm{Cr}_{2} \mathrm{O}_{3}$ in the passive film, thus improving the corrosion resistance of the base alloy. As a consequence, pitting formation was suppressed (no pitting potential was observed up to $2.0 \mathrm{~V}_{\mathrm{SCE}}$ ) and the corrosion current density decreased. The composition of the passive film was scrutinized by XPS analysis, confirming $\mathrm{Cr}_{2} \mathrm{O}_{3}$ enrichment in the passive film. This work consistently shows the importance of $\mathrm{Cr}$ addition to obtain corrosion resistant HEAs. Furthermore, tailoring of passive film composition can give a remarkable increase of resistance to pitting corrosion. The beneficial role played by $\mathrm{Cr}$ addition is the central aspect of this achievement. The third best candidate was the single-phase $\mathrm{FCC} \mathrm{CoCrFeNiGe}{ }_{0.3}$ alloy developed by Liu et al. ${ }^{75}$. The superior corrosion resistance of the Ge-containing alloy with respect to the base $\mathrm{CoCrFeNi}$ alloy was ascribed to the formation of $\mathrm{GeO}_{2}$ as part of the passive film.

From Figure 4, the ranking of corrosion resistant HEAs would proceed as follows: ${ }^{30}>77>{ }^{64}-\mathrm{b}>{ }^{73}>69>76>{ }^{17}-\mathrm{b}$. As shown in Table 2, ${ }^{30}$ is the CoCrFeNiMo ${ }_{0.4}$ alloy, ${ }^{77}$ is the $\mathrm{Ni}_{2} \mathrm{CrFeMo}_{0.2}$ alloy, ${ }^{64}$-b is the $\mathrm{CoCrFeNi}{ }_{2} \mathrm{Mo}_{0.25}$ alloy, ${ }^{73}$ is the $\mathrm{CoFeMn}_{1.2} \mathrm{NiGa}_{0.8}$ alloy, ${ }^{69}$ is the CoCrFeNiMo ${ }_{02}$ alloy, ${ }^{76}$ is the $\mathrm{Co}_{30} \mathrm{Cr}_{20} \mathrm{Fe}_{30} \mathrm{Ni}_{20}$ alloy, and ${ }^{17}-\mathrm{b}$ is the $\mathrm{CoCrFeNiAl}$ alloy. According to Shang et. ${ }^{30}$, Mo can enhance the corrosion resistance of $\mathrm{CoCrFeNiMo}{ }_{x} \mathrm{HEAs}$, depending on its content, if the formation of galvanic couples by the precipitation of Mo-rich phases is avoided. Similarly, Wei et al. ${ }^{77}$, have point to the beneficial effect of Mo to the corrosion resistance of HEAs of the $\mathrm{Ni}_{2} \mathrm{CrFeMo}_{\mathrm{x}}$, as it enhances passivity, since precipitation of Mo-rich $\sigma$-phase is restricted by proper additions

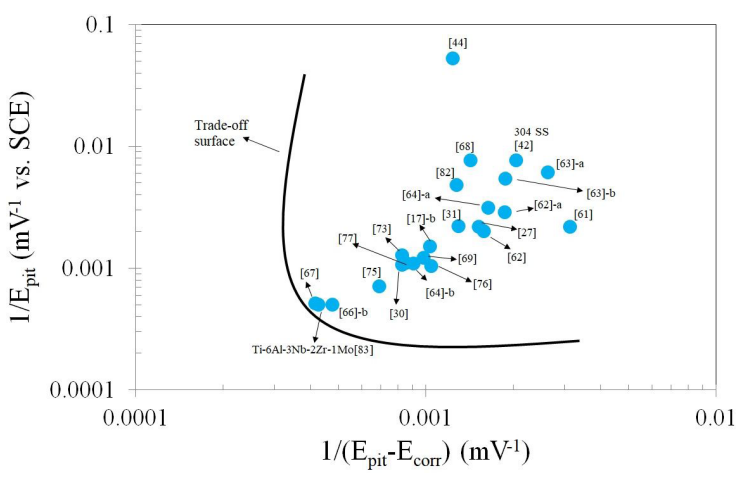

Figure 4. Trade-off plot showing $1 / \mathrm{E}_{\mathrm{pit}}$ versus $1 /\left(\mathrm{E}_{\mathrm{pit}}-\mathrm{E}_{\text {corr }}\right)$. 
of Mo to the base alloy. Rodríguez et al. ${ }^{64}$ ascribed the high corrosion resistance of the $\mathrm{CoCrFeNi}{ }_{2} \mathrm{Mo}_{0.25}$ to the formation of $\mathrm{Cr}_{2} \mathrm{O}_{3}$ and $\mathrm{MoO}_{2}$ in the passive film. Sun et al. ${ }^{73}$ explained the high corrosion resistance of the $\mathrm{CoFeMn}{ }_{1.2} \mathrm{NiGa}_{0.8}$ alloy due to homogeneous distribution of $\mathrm{Ni}$ and $\mathrm{Co}$ on the surface oxide film, forming a protective layer against corrosion. Any specific role is ascribed to $\mathrm{Ga}$ as a relevant element to the corrosion protection ability of the passive film. In reference ${ }^{69}$, the good corrosion resistance of the $\mathrm{CoCrFeNiMo}_{0.2}$ alloy is associated with the positive effect of Mo in forming a passive film with high stability. However, no further details are given about the composition of the passive film and how Mo affects the corrosion behavior of the HEA. Nascimento et al. ${ }^{17}$ explained the high corrosion resistance of the $\mathrm{CoCrFeNi}$ alloy due to the high concentration of $\mathrm{Cr}_{2} \mathrm{O}_{3}$ in the passive film.

The design criteria shown in Figure 4 can effectively drive the development of new corrosion resistant HEAs. If one bears in mind that homogeneous microstructure favors the formation of a stable passive film (single phase alloys without precipitates), then, specific passivating elements can lead to outstanding resistance to localized corrosion. The outputs of our selection process indicate that $\mathrm{Ti}, \mathrm{Nb}$ and $\mathrm{Ge}$ can greatly enhance the stability of the passive films of HEAs, provided a homogeneous microstructure is formed. Notwithstanding the usefulness of the data shown in Figure 4, it is important to highlight the lack of published data regarding the passive current densities of HEAs in sodium chloride solution. As shown in Table 2, few reports bring this information. However, it plays a pivotal role in the corrosion kinetics of passive metals. Future reports should provide this information to help consolidating materials selection criteria that could consistently drive the development of corrosion resistant HEAs. Despite this limitation, it is clear that most of the HEAs whose data are plotted in Figure 4 greatly surpass the corrosion resistance of the conventional 304 stainless steel and Ti-6Al-4V alloy, revealing the great interest that this new class of materials brings to the scientific community.

Documentation can be sought to further consolidate the design criteria established in Table 3. One further step into this direction may be realized from the bar chart shown in Figure 5. It displays the values of $\mathrm{E}_{\text {pit }}$ for selected HEAs, as collected from Table 3 . Additionally, $\mathrm{E}_{\mathrm{pit}}$ for conventional 304 stainless steel ${ }^{42}$, and for the Ti-6Al-3Nb-2Zr-1Mo ${ }^{83}$ alloy are also included for comparison purposes. The best candidates to withstand localized corrosion are those with the largest values of $\mathrm{E}_{\text {pit }}$. Careful analysis of Figure 5 allows one to reach to similar conclusions than those obtained from the trade-off plot shown in Figure 4 by showing that the most corrosion-resistant HEAs are ${ }^{66}-b,{ }^{67}$ and 75 . However, ranking does not follow the same order than that shown in Figure 4. Subtle differences can be perceived. For instance, $\mathrm{Al}_{0.3} \mathrm{Cr}_{2} \mathrm{FeCoNi}$ alloy ${ }^{66-\mathrm{b}}$ displays the largest $\mathrm{E}_{\text {pit }}$, followed by $\mathrm{TiCrVNb}_{0.5} \mathrm{Al}_{0.5}$ alloy ${ }^{67}$, which is an inverted position if one compares with the trade-off plot of Figure 4. On one hand, a more complete picture of the passive film stability is built when both criteria, $\mathrm{E}_{\mathrm{pit}}$ and $\mathrm{E}_{\text {pit }}-\mathrm{E}_{\text {corr }}$ (passive range) are considered simultaneously, as shown in Figure 4. On the other hand, Figure 5 is useful to confirm and support this interpretation, as a simple way of visualizing the performance of HEAs against localized corrosion exposed to sodium chloride solutions.

\section{Future Prospects}

Next steps towards the development of corrosion resistant HEAs must encompass the critical aspects revealed in the previous sections. The major design guidelines must rely on the basic principles of achieving an alloy with uniform distribution of alloying elements, homogeneous microstructure and compact passive films. From the materials selection procedure undertaken in the previous section, chromium is the most important individual passivating element. However, proper combinations of other passivating elements such as $\mathrm{Al}, \mathrm{Ni}, \mathrm{Mo}, \mathrm{Co}$ and Ti may improve the corrosion resistance at specific compositions. Conversely, $\mathrm{Mn}$ and $\mathrm{Cu}$ are mainly reported as having deleterious effect on the passive film stability, especially if segregations are formed. Based on these observations, a general framework of the main practical recommendations for enhancing the corrosion resistance of HEAs can be synthesized as shown in Figure 6.

In spite of the practical utility of these guidelines, this design problem is quite complex. Roy and Balasubramanian ${ }^{84}$ highlight the challenging task of designing HEAs due to the huge number of possible combinations, exceeding 590 billion, if one considers 75 different stable elements and a 10\% change in the atomic fraction of each element in HEAs with three to six elements. As a consequence, computational tools may give a valuable contribution to future studies not only devoted to new HEA formulations but also to tailor specific properties for desired applications. Recent works show the importance of integrated computational materials engineering (ICME) in the development of corrosion resistant alloys ${ }^{85,86}$. Combination of such tools with empirical knowledge of already developed materials is the next step in this dynamic research field.

In addition to support of computational tools to formulate new HEAs, it is imperative to expand the current knowledge on the corrosion behavior of these materials by further characterizing their electrochemical properties in typical corrosive environments such as acidic solutions and sodium chloride solutions. There is a lack of information regarding some electrochemical parameters, especially passive current densities. Moreover, further understanding on the correlation

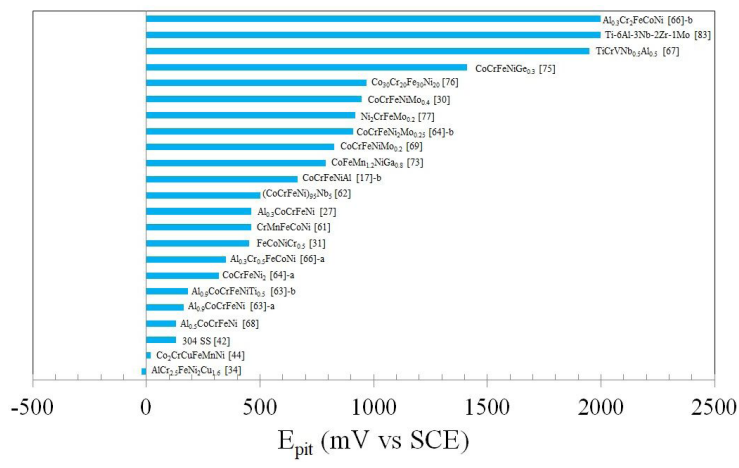

Figure 5. Bar chart showing the values of $\mathrm{E}_{\text {pit }}$ for selected HEAs in $3.5 \mathrm{wt} . \% \mathrm{NaCl}$ solution at room temperature. 
between passive film composition and corrosion properties is still needed for several HEA systems. Future experimental works should be devoted to clarifying such as aspects. Additionally, novel studies on the local electrochemical activity of multiphase HEAs are also lacking in the current literature. In this scenario, scanning high resolution probe techniques such as scanning electrochemical microscopy (SECM) should valuable information to understand the influence of different crystalline phases on the corrosion resistance of HEAs with heterogeneous microstructures. This is still an open field in the current literature.

\section{Conclusions}

The corrosion resistance of multi-principal element alloys depends on the combination of a set of attributes that are mainly related to chemical homogeneity with respect to elemental distribution through grain boundaries and crystalline phases. Moreover, passive film stability plays a central role in this regard. To meet the best performance against corrosion, careful composition control is, therefore, demanded. The Ashby method was effective at identifying the most corrosion resistant HEAs developed up to date whose corrosion properties are available in the literature. Based on the values of corrosion potential and corrosion current densities, the best candidate was $\mathrm{Co}_{2} \mathrm{Cr}_{2} \mathrm{Ni}_{2} \mathrm{AlTi}$ alloy in acidic solutions. In sodium chloride solution the design criteria were based on pitting potentials $\left(\mathrm{E}_{\mathrm{pit}}\right)$ and passive range $\left(\mathrm{E}_{\mathrm{pit}}-\mathrm{E}_{\text {corr }}\right)$, seeking for alloys with high resistance to localized corrosion. The use of a trade-off plot and penalty function $(\mathrm{Z})$ was useful to represent the corrosion data, consistently indicating the best way to rank different HEAs with respect to their resistance to pitting and/or crevice corrosion. The best candidates were $\mathrm{TiCrVNb}_{0.5} \mathrm{Al}_{0.5}$ and $\mathrm{Al}_{0.3} \mathrm{Cr}_{2} \mathrm{FeCoNi}$ alloys. Further documentation steps can give additional support to the materials selection process. In this regard, passive current densities $\left(i_{\text {pass }}\right)$ may be individually assessed with this purpose, giving a clear assessment of the anodic dissolution rate in passive metals. Likewise, pitting potentials may be represented individually as bar charts in the

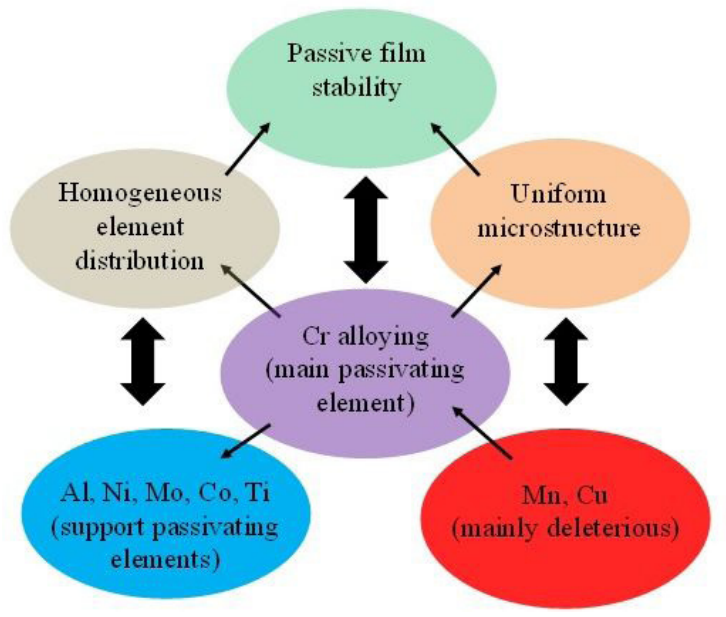

Figure 6. Schematic representation of the most important practical design guidelines for corrosion resistant HEAs. documentation step of the Ashby process, giving additional support to the information obtained in the ranking step.

The stability of the passive film depends on the alloying elements, and the formation of a homogeneous microstructure. Titanium and chromium are the most attractive passivating elements, as reported in the literature, provided microstructural and compositional homogeneities are maintained. In the same way, molybdenum can enhance the stability of the passive film of in $\mathrm{Co}$ and $\mathrm{Cr}$-containing HEAs, if galvanic couples are not formed due to the precipitation of Mo-rich phases.

\section{Acknowledgements}

Authors are grateful to the Brazilian agencies CAPES (Finance Code 001) and CNPq (Process 303604/2020-4) for the financial support.

\section{References}

1. Gómez-Esparza CD, Baldenebro-López F, González-Rodelas L, Baldenebro-López J, Martínez-Sánchez R. Series of nanocrystalline $\mathrm{NiCoAlFe}(\mathrm{Cr}, \mathrm{Cu}, \mathrm{Mo}$, Ti) high-entropy alloys produced by mechanical alloying. Mater Res. 2016;19:39-46.

2. Zhang C, Zhang F, Diao H, Gao MC, Tang Z, Polawsky JD, et al. Understanding phase stability of Al-co-Cr-Fe-Ni high entropy alloys. Mater Des. 2016;109:425-33.

3. Tsai M, Yeh J. High-entropy alloys: a critical review. Mater Res Lett. 2014;2:107-23.

4. Ikeda Y, Grabowski B, Körmann F. Ab initio phase stabilities and mechanical properties of multicomponent alloys: a comprehensive review for high entropy alloys and compositionally complex alloys. Mater Charact. 2019;147:464-511.

5. George EP, Curtin WA, Tasan CC. High entropy alloys: a focused review of mechanical properties and deformation mechanisms. Acta Mater. 2020;188:435-74.

6. Li Z, Zhao S, Ritchie RO, Meyers MA. Mechanical properties of high-entropy alloys with emphasis on face-centered cubic alloys. Prog Mater Sci. 2019;102:296-345.

7. Gludovatz B, Hohenwarter A, Catoor D, Chang EH, George EP, Ritchie RO. A fracture-resistant high-entropy alloy for cryogenic applications. Science. 2014;345:1153-8.

8. Zhang M, George EP, Gibeling JC. Tensile creep properties of a CrMnFeCoNi high-entropy alloy. Scr Mater. 2021;194:113633.

9. Brechtl J, Chen S, Lee C, Shi Y, Feng R, Xie X, et al. A review of the serrated-flow phenomenon and its role in the deformation behavior of high-entropy alloys. Metals. 2020;10:1101.

10. Chen C, Zhang H, Fan Y, Zhang W, Wei R, Wang T, et al. A novel ultrafine-grained high entropy alloy with excellent combination of mechanical and soft magnetic properties. J Magn Magn Mater. 2020;502:166513.

11. El-Atwani O, Li N, Li M, Devaraj A, Baldwin JKS, Schneider MM, Sobieraj D, Wróbel JS, Nguyen-Manh D, Maloy SA, Martinez E. Outstanding radiation resistance of tungsten-based high-entropy alloys. Sci Adv. 2019;5(3):13.

12. Shi Y, Yang B, Liaw PK. Corrosion-resistant high-entropy alloys: a review. Metals. 2017;7:43.

13. Fu Y, Li J, Luo H, Du C, Li X. Recent advances on environmental corrosion behavior and mechanism of high-entropy alloys. J Mater Sci Technol. 2021;80:217-33.

14. Qiu Y, Thomas S, Gibson MA, Fraser HL, Birbilis N. Corrosion of high entropy alloys. NPJ Mater Degrad. 2017;1:15.

15. Alaneme KK, Bodunrin MO, Oke SR. Processing, alloy composition and phase transition effect on the mechanical and corrosion properties of high entropy alloys: a review. Journal of Materials Research and Technology. 2016;5:384-93. 
16. Zheng S, Cai Z, Pu J, Zeng C, Wang L. Passivation behavior of VAlTiCrSi amorphous high-entropy alloy film with a highcorrosion resistance in artificial sea water. Appl Surf Sci. 2021;542:148520.

17. Nascimento CB, Donatus U, Ríos CT, Antunes RA. Electronic properties of the passive films formed on $\mathrm{CoCrFeNi}$ and $\mathrm{CoCrFeNiAl} \mathrm{high} \mathrm{entropy} \mathrm{alloys} \mathrm{in} \mathrm{sodium} \mathrm{chloride} \mathrm{solution.}$ Journal of Materials Research and Technology. 2020;9:1387992.

18. Shang X, Wang Z, He F, Wang J, Li J, Yu J. The intrinsic mechanism of corrosion resistance for FCC high entropy alloys. Sci China Technol Sci. 2018;61:189-96.

19. Ashby MF. Multi-objective optimization in material design and selection. Acta Mater. 2000;48:359-69.

20. Mehmood Z, Haneef I, Udrea F. Material selection for microelectro-mechanical-systems (MEMS) using Ashby’s approach. Mater Des. 2018;157:412-30.

21. Sawant VB, Mohite SS, Cheulkar LN. Comprehensive contact material selection approach for RF MEMS switch. Mater Today: Proc. 2018;5:10704-11.

22. Bird ET, Bowden AE, Seeley MK, Fullwoood DT. Materials selection of flexible open-cell foams in energy absorption applications. Mater Des. 2018;137:414-21.

23. Antunes RA, Salvador CAF, Oliveira MCL. Materials selection of optimized titanium alloys for aircraft applications. Mat Res. 2018;21(2):e20170979.

24. Antunes RA, Oliveira MCL. Materials selection for hot stamped automotive body parts: an application of the Ashby approach based on the strain hardening exponent and stacking fault energy of materials. Mater Des. 2014;63:247-56.

25. Ashby MF. Materials selection in mechanical design. 4th ed. Oxford: Elsevier; 2010.

26. Kao Y, Lee T, Chen S, Chang Y. Electrochemical passive properties of $\mathrm{Al}_{x} \mathrm{CoCrFeNi}(\mathrm{x}=0,0.25,0.50,1.00)$ alloys in sulfuric acids. Corros Sci. 2010;52:1026-34.

27. Shi Y, Yang B, Xie X, Brechtl J, Dahmen KA, Liaw PK. Corrosion of $\mathrm{Al}_{\mathrm{x}} \mathrm{CoCrFeNi}$ high-entropy alloys: al-content and potential scan-rate dependent pitting behavior. Corros Sci. 2017;119:33-45.

28. Chou YL, Yeh JW, Shih HC. The effect of molybdenum on the corrosion behaviour of the high-entropy alloys $\mathrm{Co}_{1.5} \mathrm{CrFeNi}_{1.5} \mathrm{Ti}_{0.5} \mathrm{Mo}_{x}$ in aqueous environments. Corros Sci. 2010;52:2571-81.

29. Dai C, Zhao T, Du C, Liu Z, Zhang D. Effect of molybdenum content on the microstructure and corrosion behavior of FeCoCrNiMo high-entropy alloys. J Mater Sci Technol. 2020;46:64-73.

30. Shang X, Wang Z, Wu Q, Wang J, Li J, Yu J. Effect of Mo addition on corrosion behavior of high-entropy alloys $\mathrm{CoCrFeNiMo}_{x}$ in aqueous environments. Acta Metall Engl Lett. 2019;32:41-51.

31. Chai W, Lu T, Pan Y. Corrosion behaviors of FeCoNiCr $\mathrm{Fe}_{\mathrm{x}}(\mathrm{x}=$ 0, 0,5, 1,0) multi-principal element alloys: Role of $\mathrm{Cr}$-induced segregation. Intermetallics. 2020;116:106654.

32. Chen X, Hu J, Liu Y, Xiang F. Corrosion behavior in nitric acid solution and tensile properties of $(\mathrm{CuFeNiMn})_{1-\mathrm{x}} \mathrm{Cr}_{\mathrm{x}}$ HEAs. Met Mater Int. 2021;27:2230-8.

33. Parakh A, Vaidya M, Kumar N, Chetty R, Murty BS. Effect of Crystal structure and grain size on corrosion properties of AlCoCrFeNi high entropy alloy. J Alloys Compd. 2021;863:158056.

34. Yang S, Yu W, Liu T, Li C, Zhang Y, Qu Y. Effect of Cr content on corrosion behavior of $\mathrm{AlCr}_{\mathrm{x}} \mathrm{FeNi}_{2} \mathrm{Cu}_{1.6}$ high entropy alloys. Mater Res Express. 2019;6:076501.

35. Yan X, Guo H, Yang W, Pang S, Wang Q, Liu Y et al. A10.3CrxFeCoNi high-entropy alloys with high corrosion resistance and good mechanical properties. J Alloys Compd. 2021;860:158436.

36. Wang L, Mercier D, Zanna S, Seyeux A, Laurent-Brocq $\mathrm{M}$, et al. Study of the surface oxides and corrosion behaviour of an equiatomic CoCrFeMnNi high entropy alloy by XPS and TOF-SIMS. Corros Sci. 2020;167:108507.

37. Han Z, Ren W, Yang J, Tian A, Du Y, Liu G, et al. The corrosion behavior of ultra-fine grained $\mathrm{CoNiFeCrMn} \mathrm{high-entropy} \mathrm{alloys.}$ J Alloys Compd. 2020;816:152583.

38. Karpets MV, Myslyvchenko OM, Makarenko OS, Gorban VF, Krapivka MO, Degula AI. Effect of nickel on the structure and phase composition of the $\mathrm{VCrMnFeCoNix}$ high-entropy alloy. J Superhard Mater. 2015;37:182-8.

39. Koppoju S, Konduri SP, Chalavadi P, Bonta SR, Mantripragada $\mathrm{R}$. Effect of $\mathrm{Ni}$ on microstructure and mechanical properties of $\mathrm{CrMnFeCoNi}$ high entropy alloy. Trans Indian Inst Met. 2020;73:853-62.

40. Qiu X-W, Liu C-G. Microstructure and properties of $\mathrm{Al}_{2} \mathrm{CrFeCoCuTiNi}{ }_{x}$ high-entropy alloys prepared by laser cladding. J Alloys Compd. 2013;553:216-20.

41. Ríos ML, Perdomo PPS, Voiculescu I, Geanta V, Crãciun V, Boerasu I et al. Effects of nickel contente on the microstructure, microhardness and corrosion behavior of high-entropy AlCoCrFeNix alloys. Sci Rep. 2020;10:21119.

42. Huang L, Wang X, Zhao X, Wang C, Yang Y. Analysis on the key role in corrosion behavior of CoCrNiAlTi-based high entropy alloy. Mater Chem Phys. 2021;259:124007.

43. Qiu X-W. Corrosion behavior of $\mathrm{Al}_{2} \mathrm{CrFeCo}_{\mathrm{x}} \mathrm{CuNiTi}$ highentropy alloy coating in alkaline solution and salt solution. Results in Physics. 2019;12:1737-41.

44. Zhao R-F, Ben B, Cai B, Liu Z-X, Zhang G-P, Zhang J-J. Corrosion behavior of $\mathrm{Co}_{\mathrm{x}} \mathrm{CrCuFeMnNi}$ high-entropy alloys prepared by hot pressing sintered in $3.5 \% \mathrm{NaCl}$ solution. Results in Physics. 2019;15:102667.

45. Wu P, Gan K, Yan D, Fu Z, Li Z. A non-equiatomic FeNiCoCr high-entropy alloy with excellent anti-corrosion performance and strength-ductility synergy. Corros Sci. 2021;183:109341.

46. Torbati-Sarraf H, Shabani M, Jablonski PD, Pataky GJ, Poursaee A. The influence of incorporation of $\mathrm{Mn}$ on the pitting corrosion performance of $\mathrm{CrFeCoNi}$ high entropy alloy at different temperatures. Mater Des. 2019;184:108170.

47. Wong S, Shun T, Chang C, Lee C. Microstructures and properties of $\mathrm{Al}_{0.3} \mathrm{CoCrFeNiMn}_{\mathrm{x}}$ high-entropy alloys. Mater Chem Phys. 2018;210:146-51.

48. Zhang YS, Zhu XM. Electrochemical polarization and passive film analysis of austenitic Fe-Mn-Al steels in aqueous solutions. Corros Sci. 1999;41:1817-33.

49. Sahu S, Swanson OJ, Li T, Gerard AY, Scully JR, Franekl GS. Localized corrosion behavior of non-equiatomic $\mathrm{NiFeCrMnCo}$ multi-principal element alloys. Electrochim Acta. 2020;354:136749.

50. Abolkassem SA, Mohamed LZ, Gaber GA, Elkady OA. Microstructure and corrosion behavior of $\mathrm{FeNiCoCrCu}$ and FeNiCoCrMn high entropy alloys manufactured by powder metallurgy in different acid media. Journal of Materials Research and Technology. 2021;10:1122-42.

51. Zheng H, Chen R, Qin G, Li X, Su Y, Ding H, et al. Microstructure evolution, $\mathrm{Cu}$ segregation and tensile properties of $\mathrm{CoCrFeNiCu}$ high entropy alloy during directional solidification. J Mater Sci Technol. 2020;38:19-27.

52. Campo KN, Freitas CC, Fanton L, Caram R. Melting behavior and blobular microstructure formation in semi-solid $\mathrm{CoCrCuxFeNi}$ high-entropy alloys. J Mater Sci Technol. 2020;52:207-17.

53. Nene SS, Frank M, Liu K, Sinha S, Mishra RS, McWilliams BA, et al. Corroion-resistant high entropy alloy with high strength and ductility. Scr Mater. 2019;166:168-72.

54. Scully JR, Inman SB, Gerard AY, Taylor CD, Windl W, Schreiber DK, et al. Controlling the corrosion resistance of multi-principal element alloys. Scr Mater. 2020;188:96-101.

55. Xiao DH, Zhou PF, Wu WQ, Diao HY, Gao MC, Song $\mathrm{M}$, et al. Microstructure, mechanical and corrosion behaviors of AlCoCuFeNi-(Cr,Ti) high entropy alloys. Mater Des. 2017;116:438-47. 
56. Yen C, Lu H, Tsai M, Wu B, Lo Y, Wang C, et al. Corrosion mechanism of annealed equiatomic $\mathrm{AlCoCrFeNi}$ tri-phase high-entropy alloy in $0.5 \mathrm{M} \mathrm{H}_{2} \mathrm{SO}_{4}$ aerated aqueous solution. Corros Sci. 2019; 157:462-71.

57. Fu Y, Dai C, Luo H, Li D, Du C, Li X. The corrosion behavior and film properties of Al-containing high-entropy alloys in acidic solutions. Appl Surf Sci. 2021;560:149854.

58. Zhang JJ, Yin XL, Dong Y, Lu YP, Jiang L, Wang TM et al. Corrosion properties of AlxCoCrFeNiTi0.5 high entropy alloys in 0.5 M H2SO4 aqueous solution. Mater Res. 2014;18(suppl. 4):S4756-S4759.

59. Yang J, Wu J, Zhang CY, Zhang SD, Yang BJ, Emori W, et al. Effects of $\mathrm{Mn}$ on the electrochemical corrosion and passivation behavior of $\mathrm{CoFeNiMnCr}$ high-entropy alloy system in $\mathrm{H}_{2} \mathrm{SO}_{4}$ solution. J Alloys Compd. 2020;819:152943.

60. Sun YP, Wang Z, Yang HJ, Lan AD, Qiao JW. Effects of the element $\mathrm{La}$ on the corrosion properties of $\mathrm{CrMnFeNi}$ high entropy alloys. J Alloys Compd. 2020;842:155825.

61. Ye Q, Feng K, Li Z, Lu F, Li R, Huang J, et al. Microstructure and corrosion properties of $\mathrm{CrMnFeCoNi}$ high entropy alloy coating. Appl Surf Sci. 2017;396:1420-6.

62. Wang W, Qi W, Xie L, Yang X, Li J, Zhang Y. Microstructure and corrosion behavior of $(\mathrm{CoCrFeNi})_{95} \mathrm{Nb}_{5}$ high-entropy alloy coating fabricated by plasma spraying. Materials. 2019;12:694.

63. Qiu Y, Thomas S, Fabijanic D, Barlow AJ, Fraser HL, Birbilis N. Microstructural evolution, electrochemical and corrosion properties of $\mathrm{Al}_{\mathrm{x}} \mathrm{CoCrFeNiTi}_{\mathrm{y}}$ high entropy alloys. Mater Des. 2019;170:107698.

64. Rodriguez AA, Tylczak JH, Gao MC, Jablonski PD, Detrois M, Ziomek-Moroz M, et al. Effect of molybdenum on the corrosion behavior of high-entropy alloys $\mathrm{CoCrFeNi}_{2}$ and $\mathrm{CoCrFeNi} \mathrm{Mo}_{0.25}$ under sodium chloride aqueous solutions. Adv Mater Sci Eng. 2018;2018:3016304.

65. Wang H, Liu J, Xing Z, Ma G, Cui X, Jin G, et al. Microstructure and corrosion behaviour of AlCoFeNiTiZr high-entropy alloy films. Surf Eng. 2020;36(1):78-85

66. Yan X, Guo H, Wang W, Pang S, Wang Q, Liu Y, et al. ${ }_{0.3} \mathrm{Cr}_{\mathrm{X}} \mathrm{FeCoNi}$ high-entropy alloys with high corrosion resistance and good mechanical properties. J Alloys Compd. 2021;860:158436.

67. Li M, Chen Q, Cui X, Peng X, Huang G. Evaluation of corrosion resistance of the single-phase light refractory high entropy alloy $\mathrm{TiCrVNb}_{05} \mathrm{Al}_{05}$ in chloride environment. J Alloys Compd. 2020;857:158278

68. Lin C, Tsai H. Evolution of microstructure, hardness, and corrosion properties of high-entropy $\mathrm{Al}_{0.5} \mathrm{CoCrFeNi}$ alloy. Intermetallics. 2011;19:288-94.

69. Wang Q, Amar A, Jiang C, Luan H, Zhao S, Zhang H, et al. $\mathrm{CoCrFeNiMo}_{02}$ high entropy alloy by laser melting deposition: prospective material for low temperature and corrosion resistant applications. Intermetallics. 2020;119:106727.

70. Wang W, Wang J, Sun Z, Li J, Li L, Song X, et al. Effect of Mo and aging temperature on corrosion behavior of $(\mathrm{CoCrFeNi})_{100-x} \mathrm{Mo}_{x}$ high-entropy alloys. J Alloys Compd. 2020;812:152139.
71. Yu Y, Xu N, Zhu S, Qiao Z, Zhang J, Yang J, et al. A novel $\mathrm{Cu}$-coped high entropy alloy with excellent comprehensive performances for marine application. J Mater Sci Technol. 2021;69:48-59.

72. Muangtong P, Rodchanarowan A, Chaysuwan D, Chanlek N, Goodall R. The corrosion behaviour of CoCrFeNi-x $(x=\mathrm{Cu}$, Al, Sn) high entropy alloy. Corros Sci. 2020;172:108740.

73. Sun X, Du L, Lan H, Cui J, Wang L, Li R, et al. Mechanical, corrosion and magnetic behavior of a CoFeMn ${ }_{1.2} \mathrm{NiGa}_{0.8}$ high entropy alloy. J Mater Sci Technol. 2021;73:139-134.

74. Sünbul SE, Için K, Seren FZ, Sahin O, Çakil DD, Sezer R, et al. Determination of structural, isothermal oxidation and corrosion properties of Al-Co-Cr-Fe-Ni-Ti-Cu high-entropy alloy. Vacuum. 2021;187:110072

75. Liu H, Tsai C. Effect of Ge addition on the microstructure, mechanical properties, and corrosion behavior of $\mathrm{CoCrFeNi}$ high-entropy alloys. Intermetallics. 2021;132:107167.

76. Yang H, Shang X, Wang L, Wang Z, Wang J, Lin X. Effect of constituent elements on the corrosion resistance of singlephase $\mathrm{CoCrFeNi}$ high-entropy alloys in $\mathrm{NaCl}$ solution. Chin Shu Hsueh Pao. 2018;54:905-10. [in Chinese]

77. Wei L, Wu Q, Shang X, Li J, Wang J. Effect of Mo element and heat treatment on corrosion resistance of $\mathrm{Ni}_{2} \mathrm{CrFeMo}_{x}$ high-entropy alloy in $\mathrm{NaCl}$ solution. Chin Shu Hsueh Pao. 2019;55:840-8. [in Chinese]

78. Saifi H, Bernard MC, Joiret S, Rahmouni K, Takenouti H, Talhi B. Corrosion inhibitive action of cysteine on $\mathrm{Cu}-30 \mathrm{Ni}$ alloy in aerated 0.5 $\mathrm{M} \mathrm{H}_{2} \mathrm{SO}_{4}$. Mater Chem Phys. 2010;120:661-9.

79. Wang Q, Huang F, Cui Y-T, Yoshida H, Wen L, Jin Y. Influences of formation potential on oxide film of TC4 in $0.5 \mathrm{M}$ sulfuric acid. Appl Surf Sci. 2021;544:148888

80. Monaco L, Avramovic-Cingara G, Palumbo G, Erb W. Corrosion behaviour of electrodeposited nanocrystalline nickel-iron (NiFe) alloys in dilute $\mathrm{H}_{2} \mathrm{SO}_{4}$. Corros Sci. 2018;130:103-12.

81. Duca D, Dan ML, Vaszilcsin N. Ceftriaxone as corrosion inhibitor for nickel in acid solutions. AEF. 2018;27:74-82.

82. Zhao P, Song Y, Dong K, Shan D, Han E-H. Corrosion behavior of dual-phase Ti-6Al-4V alloys: A discussion on the impact of Fe content. J Alloys Compd. 2021;858:157708

83. Su B, Luo L, Wang B, Su Y, Wang L, Ritchie RO, et al. Annealed microstructure dependent corrosion behavior of Ti-6Al-3Nb2Zr-1Mo alloy. J Mater Sci Technol. 2021;62:234-48.

84. Roy A, Balasubramanian G. Predictive descriptors in machine learning and data-enabled explorations of high-entropy alloys. Comput Mater Sci. 2021;193:110381.

85. Lu P, Saal JE, Olson GB, Li T, Swanson OJ, Frankel GS, et al. Computational materials design of a corrosion resistant high entropy alloy for harsh environments. Scr Mater. 2018;153:19-22.

86. Taylor CD, Lu P, Saal J, Frankel GS, Scully JR. Integrated computational materials engineering of corrosion resistant alloys. NJP Mater Degrad. 2018;2:6 